\title{
A geometric treatment of Jellett's egg
}

\author{
Nawaf M. Bou-Rabee ${ }^{1, *}$, Jerrold E. Marsden ${ }^{2, * *}$, and Louis A. Romero ${ }^{3, * * *}$ \\ ${ }^{1}$ Applied \& Computational Mathematics, Caltech, Pasadena, CA 91125 \\ 2 Control \& Dynamical Systems, Caltech, Pasadena, CA 91125 \\ ${ }^{3}$ Sandia National Laboratories, P.O. Box 5800, MS 1110, Albuquerque, NM 87185-1110
}

Received 22 April 2004, accepted 23 November 2004

Published online 10 June 2005

Key words rising egg, dissipation-induced instability, Jellett momentum map, adiabatic invariants MSC (2000) 70E18, 70F40, 34D23, 34E13, 37J15, 37M05, 37N05

\begin{abstract}
This paper explains and gives a global analysis of the "rising egg" phenomenon. The main tools that are used in this analysis are derived from the theory of dissipation-induced instabilities, adiabatic invariants, and LaSalle's invariance principle. The analysis is done within the framework of a specific model of the egg as a prolate spheroid, with its equations of motion derived from Newtonian mechanics. The paper begins by considering the linear and nonlinear stability of the non-risen and risen states of the spheroid corresponding to the initial and final state of the rising egg phenomenon. The asymptotic state of the spheroid is determined by an adiabatic momentum invariant. Because the symmetry associated with this adiabatic invariant coincides with the symmetry associated with the Jellett invariant in the tippe top, we call this quantity the Jellett momentum map. Linear theory shows that the spectral stability of the non-risen state is determined by a cubic polynomial. The spectral stability of the risen state is governed by the modified Maxwell-Bloch equations - a normal form that appears in the problem of tippe top inversion and that was studied previously by the authors. A generalization of the energy-momentum method that includes adiabatic momentum invariants provides explicit criteria for the existence of an orbit connecting these states. In particular, it is shown that if the risen state is stable, the spheroid rises all the way.
\end{abstract}

(c) 2005 WILEY-VCH Verlag GmbH \& Co. KGaA, Weinheim

\section{Introduction}

The "rising egg" phenomenon manifests itself most clearly when one spins a hard-boiled egg with its long axis horizontal. If spun sufficiently fast, the egg rises from it's non-risen state to a risen state as shown in Fig. 1. The egg remains in the risen state until its rotational motion slows down due to frictional torque. Since the effect of frictional torque is not essential to the rising phenomenon (its main effect is to retard the rotational motion of the spinning egg), the model presented in this paper neglects frictional torque or torsional damping; as a consequence, the non-risen and risen states become steady-state phenomena. However, frictional torque will need to be taken into account to, for example, have a higher fidelity simulation or develop mechanisms to sustain the risen state - as the Perpetuator ${ }^{\circledR}$ for the Levitron ${ }^{\circledR}$ does. (See Hones et al. [10] and Harrigan [8] for more details.)

In this paper the rising egg phenomenon is explained through a combination of dissipation-induced instabilities, adiabatic invariants, and LaSalle's invariance principle. A dissipation-induced instability describes a neutrally stable equilibrium at an energetic saddle point (a situation that is sometimes called a gyroscopically stabilized state) becoming spectrally unstable (and hence nonlinearly unstable) with the addition of dissipation. Dissipation induced instabilities itself has a long history, which goes back to Thomson (Lord Kelvin) and Tait [19]. In its modern form, it was shown to both be a general phenomenon for gyroscopically stabilized systems and to provide a sharp converse to the energy momentum stability method by Bloch, Krishnaprasad, Marsden, and Ratiu [2,3]. We refer the reader to these papers for additional examples and the basic theory.
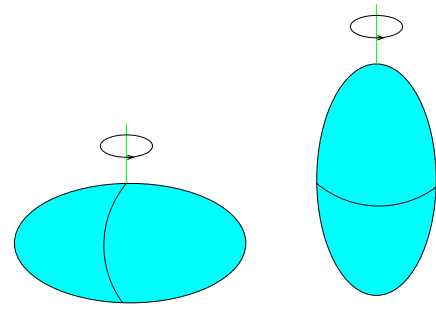

Fig. 1 L-R: a sketch of the non-risen and risen states of the symmetric egg, which is approximated as a spheroid.

* Corresponding author, e-mail: nawaf@acm.caltech.edu

** e-mail: marsden@cds.caltech.edu

*** e-mail: Iromero@sandia.gov 
By considering the rising egg as a dissipation-induced instability, one observes precisely how it relates to the wide range of dissipation-induced instabilities in other physical systems. In particular, this view affords a direct comparison to a closely related phenomenon: tippe top inversion (cf. Bou-Rabee et al. [4]). That paper treats the tippe top as a ball with an eccentric center of mass and hereafter tippe top inversion will refer to the ball with eccentric center of mass rising from a gravitationally favorable position to an unfavorable one because of dissipation.

The key difference between the egg and the tippe top is the following. The tippe top, being modeled with a spherical base, has a symmetry through the point of contact, which leads to a conservation law, the Jellett invariant. The egg, which is modeled as a spheroid, has only an approximate symmetry because of its ellipticity. Thus, in the case of the egg, one needs to deal not with invariants, but with adiabatic invariants, a key difference dealt with in references discussed below and in the present paper.

In the remainder of this section the history of the rising egg problem is discussed and the main goals and organization of the paper are laid out.

History. Since the turn of the twentieth century at least, physicists understood that the rising of spinning bodies was fundamentally related to dissipative effects, see e.g. Crabtree [5] and Routh [16]. Subsequent mathematical work focused primarily on spherical approximations of a tippe top. See Bou-Rabee et al. [4] for a survey of works on tippe top inversion.

The first serious attempt at directly understanding the motion of a symmetric egg was conducted by Moffatt and Shimomura [13]. They correctly identify and study the highest-order effects in the dynamics of a symmetric egg. Their work is noteworthy in this respect, although it did leave some doubts; see Ruina [17]. Specifically, it was not clear from Moffatt and Shimomura's work how the rising egg related to the tippe top and how valid their assumptions were.

The work of Sasaki [18] nicely extends the work of Moffatt and Shimomura [13] to more general geometries, but applies the same basic theory. The more recent work of Moffatt, Shimomura, and Branicki [14], kindly made available to the authors while this paper was in review, fills in some of the technical details left out of Moffatt and Shimomura [13]. The main contribution of these works is the discovery of the adiabatic invariance of the Jellett momentum map. This discovery was an important ingredient in the analysis of the global aspects of the rising egg phenomenon given in the present paper. Subsequent work considered the very interesting possibility that the surface normal reaction force could change sign, implying that the egg jumps, see Shimomura, Branicki, and Moffatt [15].

However, a complete mathematical model and explanation of the rising of an egg has been lacking and its obvious ties to the tippe top not fully explored. This paper fills this gap by extending what is known about the tippe top to explain the rising egg phenomenon.

Main goals of paper. This paper provides a mathematical model, analysis and simulation of the rising egg phenomenon. The main accomplishments are: 1) an extension of the normal form for tippe top inversion, namely the modified Maxwell-Bloch equations, to the rising egg, 2) completion of the linear and global nonlinear analysis of the rising egg, and 3) introduction of the energy-adiabatic momentum method through this example. Since all first integrals are trivially adiabatic invariants, the energy-adiabatic momentum method applies to a larger set of problems than the standard energy-momentum method. In future work, we hope to clarify the general method's applicability and limitations.

The egg is modelled as a spheroid (an ellipsoid with one axis of symmetry). The paper starts with a discussion of the geometry of the spheroid followed by a standard derivation of the dimensional equations of motion using Newtonian mechanics. It should be emphasized that because a sliding friction law is used, the problem is holonomically constrained - an observation which considerably simplifies the description of the dynamics. A dimensional analysis reduces the eight physical parameters in the problem to four dimensionless parameters: dimensionless mass, inertia ratio, aspect ratio, and Froude number - a dimensionless measure of how fast the top is spinning compared to the gravitational time-scale. In the last section, simulations, which can be downloaded from http://www. acm. caltech. edu/ nawaf/risingegg.html, are given in support of the analytical results.

The nonlinear analysis for the tippe top was carried out using the Jellett invariant, which reduced the dynamics to its level sets in phase space. The Jellett invariant, $\mathbf{J}$, is defined as the angular momentum, $\mathbf{L}$, projected on the vector $\mathbf{Q}$, connecting the center of mass of the tippe top to the contact point, i.e.,

$$
\mathbf{J}=\mathbf{L} \cdot \mathbf{Q}
$$

The Jellett invariant is the momentum map for the $\mathbb{S}^{1}$ action on the configuration space of the tippe top, $Q=\operatorname{SO}(3) \times \mathbb{R}^{2}$, and which can be computed in standard fashion; for example, by formula (12.2.1) of Marsden and Ratiu [12]. This momentum is no longer conserved for the spheroid. In fact, with an appropriate definition of the Jellett quantity, it results in a strict constant of the motion for axisymmetric rigid bodies on a flat surface if and only if the body geometry is spherical (see the Appendix). 
However, under a fast top approximation, the Jellett momentum $\mathbf{J}$ is an adiabatic invariant in rising egg motion. The procedure to prove this is a simple form of averaging which introduces an $S^{1}$ symmetry corresponding to the symmetry of the Jellett conservation law observed in the tippe top. Thus, this adiabatic invariant will be called the Jellett momentum map. The presence of this adiabatic invariant is critical to the nonlinear analysis in this paper as well as to an intuitive understanding of the rising egg phenomenon. It is shown that under a fast top approximation, $\epsilon \ll 1$, (where $\epsilon$ can be thought of as being inversely related to the spin rate) this momentum is constant with $O\left(\epsilon^{2}\right)$ error on the time-scale $t / \epsilon$. Since the spheroid rises only if the spin is fast enough, this fast-top approximation is appropriate.

Moreover, an argument similar to the one used in the Appendix to show that there is a strict Jellett invariant in the motion of axisymmetric rigid bodies on a flat surface if and only if the body geometry is spherical can be used to show that the Jellett momentum map is an adiabatic invariant in all axisymmetric rigid bodies. Thus, the basic theory laid out in this paper should explain the rising of a larger class of axisymmetric rigid bodies such as oblate spheroids, pear-shaped tops, and asymmetric eggs.

Linear theory applied to the equations of motion readily reveals the criterion for stability of the non-risen state. The non-risen state is not a fixed point of the derived equations of motion. However, in rotating coordinates the periodic solution corresponding to the non-risen state becomes a fixed point of the transformed system. Subsequent reduction by translational symmetry, reduces the stability of the non-risen state to the analysis of a cubic polynomial in the nutation angle with standard damping, rotational damping, gyroscopic, and gravitational parameters. The rotational damping term is not a frictional torque term, i.e., it is a moment due to the sliding frictional force and not torsional damping; it depends on the spin rate and a dimensionless friction factor that is linearly related to the nutation angle, while standard damping depends on just the dimensionless friction factor that is linearly related to the time rate of change of the nutation angle. Reasons are provided for why neglecting translational effects is valid, and the effect of each of these terms is explored. In particular, it is shown that rotational damping is critical to explaining the egg rising phenomenon, i.e., rotational damping is the source of the instability in the gravitationally stable non-risen state and the source of stability in the gyroscopically stable risen state.

In the fast-spin approximation, the risen state is explicitly derived. It is shown that the risen state is actually plural in the sense that the axis of symmetry could be pointing up or down but, the angular momentum corresponding to these states is the same. Moreover, the stability of these states is governed by the modified Maxwell-Bloch equations found in the analysis of the tippe top.

An energy landscape argument applied to the level sets of the Jellett adiabatic invariant reveals all possible minimizers of the energy. The energy's orbital derivative is shown to be negative semi-definite, and linearly related to the norm of the slip velocity by a dimensionless friction factor. Thus, asymptotic states are characterized by no-slip and no-force. The no-force modifier is added to distinguish this asymptotic state from a no-slip, nonholonomic problem. The no-slip, no-force state is a Lagrange top with solutions defined by a nutation angle (orientation) and spin rate. Reasonably, one can assume that the asymptotic state corresponds to a no-slip, no-force problem which minimizes the energy subject to the constraint of constant Jellett momentum map. This energy-adiabatic momentum method determines when these minimizers include only the asymptotically stable risen and asymptotically unstable non-risen states. Under these conditions a slight modification of LaSalle's invariance principle to take into account the adiabatic invariance of the Jellett momentum map implies a connection between these states in phase space. We note in passing that a similar global connecting argument was given in Jalnapurkar and Marsden [7] in the context of mechanical systems with symmetry and internal damping.In particular, it is shown if the risen state is asymptotically stable, the spheroid rises all the way. Moreover, the linear and nonlinear theory explicitly agree; a notable similarity to the nonlinear analysis of tippe top inversion given in Bou-Rabee et al. [4].

When the risen and non-risen states are asymptotically unstable, a tumbling (precession, spin, and no nutation) or stationary solution minimizes the energy subject to the constraint of constant Jellett momentum map. The latter happens when the spheroid cannot rise to a position which minimizes energy and instead wobbles until sliding friction dissipates all of its kinetic energy and the spheroid comes to rest.

Assuming the Jellett momentum map $\mathbf{J}$ given by (1) is constant, a simpler energy argument can intuitively explain the egg rising phenomenon. Consider the two energy states of the spheroid corresponding to the non-risen and risen states. In the non-risen/risen states, the contact vector points in the vertical and has smallest/largest magnitude respectively. Thus, the gravitational energy of the non-risen state is smaller than that of the risen state. However, assuming $\mathbf{J}$ is constant, the rotational kinetic energy of the non-risen state is larger than the rotational kinetic energy of the risen state. If the spin is fast enough, this drop in rotational kinetic energy overwhelms the increase in gravitational potential energy. Thus, when the egg rises all the way, the point which minimizes total energy is the risen state where the gravitational potential energy is greatest. As mentioned earlier fast-spin also implies the Jellett momentum map is nearly invariant. Since rising is only possible with fast-spin, the Jellett momentum map is always nearly invariant whenever rising happens, and therefore, this intuitive explanation can be expected to hold when rising happens. 
Organization. In Sect. 2, the dimensionless equations of motion for a spheriod moving on a flat surface are derived using Newtonian mechanics. Moreover, it is shown that the nonlinear equations of motion averaged over the fast dynamics conserve the averaged Jellett momentum map. In Sect.3, the equations are linearized about the non-risen state in a straightforward way. Reduction by translational symmetry, then reduces the linear equations to a system with three degrees of freedom. The stability of this system is studied in detail within the section and in the Appendix.

In Sect. 4, the risen state is derived assuming that the Jellett momentum map is nearly constant. The equations linearized about the risen state are shown to be of the form of the modified Maxwell-Bloch equations.

In Sect.5, egg rising is explained from an energy landscape perspective. A straightforward application of LaSalle's Invariance principle determines the dynamics of the asymptotic state of the spheroid. An energy-adiabatic momentum method is used to determine all possible minimizers of the energy on a level set of the Jellett momentum map, i.e., subject to an adiabatic momentum constraint. Based on this analysis, explicit criteria are provided for the existence of an orbit connecting the risen and non-risen states.

In Sect. 6, simulation data are given and results discussed. These results verify the local and global analysis in the paper. Finally in Sect. 7 possible extensions of these results to the rattleback are discussed.

\section{Equations of motion}

This section contains a standard derivation of the equations of motion for a symmetric egg on a surface; we include this standard material for the reader's convenience.

Spheroid geometry. We will model a symmetric egg by a spheroid surface in $\mathbb{R}^{3}$, namely by a the level-set of the form:

$$
\frac{x^{2}+y^{2}}{a^{2}}+\frac{z^{2}}{b^{2}}=1 .
$$

Introduce the aspect ratio $\alpha=a / b$ to rewrite the equation of a spheroid as

$$
x^{2}+y^{2}+\alpha^{2} z^{2}=a^{2} .
$$

Assume that $0<\alpha \leq 1$, which means that the spheroid is prolate, i.e., a spheroid with polar radius $b$ and equatorial radius $0<a \leq b$. Let $\mathbf{i}, \mathbf{j}, \mathbf{k}$ be the principal axes with respect to the geometric center of the spheroid and $\mathbf{k}$ the symmetry axis. Let $\mathbf{x}=\left(x_{1}, x_{2}, x_{3}\right)$ represent a vector in $\mathbb{R}^{3}$. Then (2) can be written in vector notation as:

$$
\phi(\mathbf{x}):=(\mathbf{x} \cdot \mathbf{x})+(\mathbf{x} \cdot \mathbf{k})^{2}\left(\alpha^{2}-1\right)=a^{2}
$$

or, using matrix notation to represent the quadratic form, as:

$$
\phi(\mathbf{x})=\mathbf{x}^{T}\left(\mathbf{I}+\left(\alpha^{2}-1\right) \mathbf{k} \mathbf{k}^{T}\right) \mathbf{x}=a^{2} .
$$

Contact geometry. Let $\mathbf{e}_{z}$ represent the unit vector in the direction of the vertical. For any given body orientation, $\mathbf{k}$, the contact vector with respect to the geometric center of the body is the point on the body with minimum "height". More concretely, the contact vector, $\mathbf{Q}$, minimizes $\mathbf{x} \cdot \mathbf{e}_{z}$ subject to the constraint $\phi(\mathbf{x})=a^{2}$. Equivalently, $\mathbf{Q}$ is a critical point of the function

$$
h(\mathbf{x})=\mathbf{x} \cdot \mathbf{e}_{z}+\lambda \phi(\mathbf{x}),
$$

where $\lambda$ is a Lagrange multiplier. The critical point of $h(\mathbf{x})$ solves

$$
\mathbf{e}_{z}-\frac{\mathbf{x} \cdot \mathbf{e}_{z}}{a^{2}}\left(\mathbf{x}+(\mathbf{x} \cdot \mathbf{k})\left(\alpha^{2}-1\right) \mathbf{k}\right)=0
$$

and the solution is:

$$
\mathbf{Q}=\frac{-b}{\left(\alpha^{2}+\left(1-\alpha^{2}\right)\left(\mathbf{e}_{z} \cdot \mathbf{k}\right)^{2}\right)^{1 / 2}}\left(\alpha^{2} \mathbf{e}_{z}+\left(1-\alpha^{2}\right)\left(\mathbf{e}_{z} \cdot \mathbf{k}\right) \mathbf{k}\right) .
$$

For $\theta \in[0,2 \pi)$ and $\mathbf{k}=(\cos (\theta), \sin (\theta), 0)$, the locus of points $\mathbf{q}(\mathbf{k}(\theta))$ generates is shown in Fig. 2 .

Dimensional equations of motion. In the following bold denotes vectors and no bold denotes points (e.g., the vector $\mathbf{Q}$ and the point $Q$ ). The equations of motion for a spheroid with polar radius $b$, mass $M$, and exactly one point $Q$ on a fixed horizontal plane for all times will be derived here (see Fig. 3). 

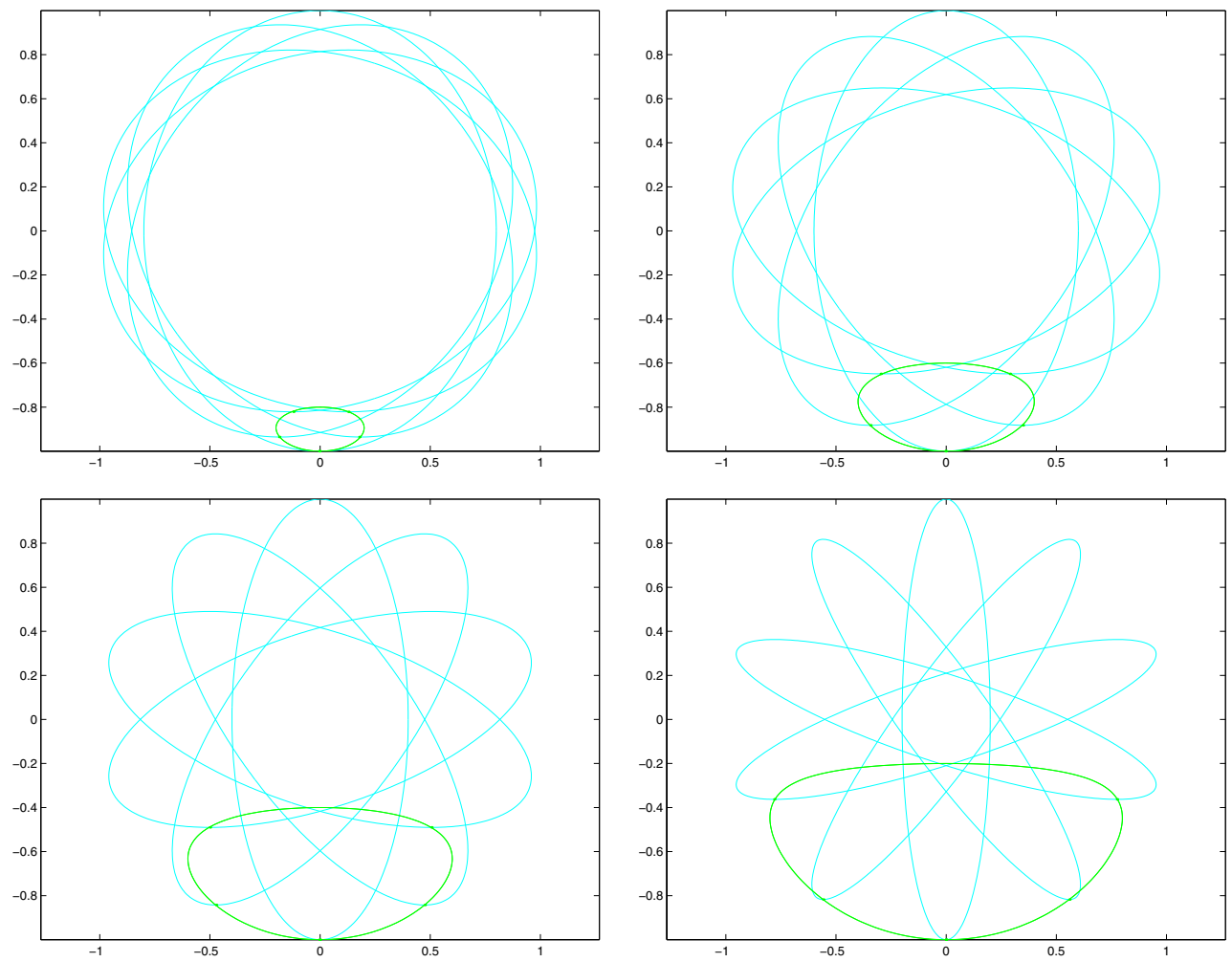

Fig. 2 The locus of contact points (green) with some corresponding ellipses (cyan) in the plane for $\alpha=0.8,0.6,0.4,0.2$, $b=1$, and $\mathbf{k}=(\cos (\theta), \sin (\theta), 0)$ where $0 \leq \theta \leq 2 \pi$.

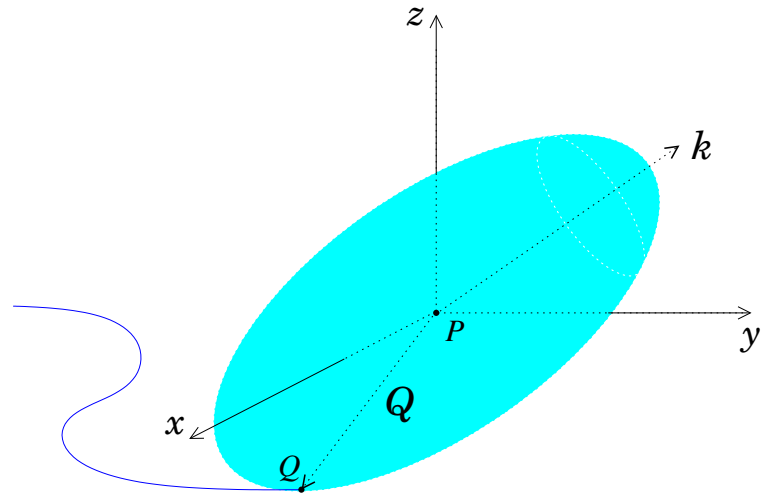

Fig. 3 The spheroid on a plane with geometric center $P$ (also the center of mass) and contact point $Q$. Vectors $\mathbf{q}$ and $\mathrm{k}$ represent the dimensionless position of the contact point with respect to the center of mass and the unit vector in the direction of the axis of symmetry respectively.

The fixed horizontal plane is defined to be the set of all points $\mathbf{x} \in \mathbb{R}^{3}$ such that $\mathbf{x} \cdot \mathbf{e}_{z}=0$. A force $\mathbf{F}_{Q}$ acting at $Q$ is assumed and will be specified towards the end of the section. The position of the geometric center of the spheroid for any dimensional time $T$ is given by the coordinates $P(T)=(X(T), Y(T), Z(T))$ for $T \in[0, \infty)$ such that $X(0)=Y(0)=0$ and $\alpha b=a \leq Z(0)=$ constant $\leq b$. The inertial frame is coordinatized by unit vectors $\mathbf{e}_{\mathbf{x}}, \mathbf{e}_{\mathbf{y}}, \mathbf{e}_{z}$. The orientation of the body at any time $T$ is given by the axis of symmetry $\mathbf{k}(T)$. We assume the mass distribution is symmetric with respect to $\mathbf{k}(T)$ which implies the moments of inertia about the principal axes $\mathbf{i}(T)$ and $\mathbf{j}(T)$ with respect to $P(T)$ are equal, i.e., $I_{i}=I_{j}=I$. The angular velocity and angular momentum of the body with respect to $P(T)$ are given by $\boldsymbol{\omega}(T)$ and $\mathbf{L}(T)$.

Balance of linear momentum yields:

$$
\begin{aligned}
M \ddot{X} & =\mathbf{F}_{Q} \cdot \mathbf{e}_{x}, \\
M \ddot{Y} & =\mathbf{F}_{Q} \cdot \mathbf{e}_{y}, \\
M \ddot{Z} & =\mathbf{F}_{Q} \cdot \mathbf{e}_{z}-M g,
\end{aligned}
$$


while balance of angular momentum gives

$$
\dot{\mathbf{L}}=\mathbf{Q} \times \mathbf{F}_{Q}
$$

and the evolution of the axis of symmetry is just by advection as usual:

$$
\dot{\mathrm{k}}=\omega \times \mathbf{k}
$$

where $\boldsymbol{\omega}$ can be expressed in terms of the angular momentum as:

$$
\boldsymbol{\omega}=\frac{1}{I}\left(\mathbf{L}+\frac{\left(I-I_{k}\right)(\mathbf{L} \cdot \mathbf{k})}{I_{k}} \mathbf{k}\right)
$$

where $I_{k}$ and $I=I_{j}=I_{i}$ are the moments of inertia with respect to the axis of symmetry and the other principal axes respectively. Therefore,

$$
\dot{\mathbf{k}}=\frac{1}{I} \mathbf{L} \times \mathbf{k} .
$$

The constraint that the spheroid remains on the flat surface is expressed as

$$
\mathbf{Q} \cdot \mathbf{e}_{z}+Z=0 \text {. }
$$

Contact force. The force exerted on the body at the contact point $Q$ is due to surface friction and normal reaction: $\mathbf{F}_{Q}=$ $\mathbf{F}_{f}+F_{z} \mathbf{e}_{z}$. We assume the surface frictional force is proportional to the slip velocity, i.e., the velocity of the contact point on the rigid body relative to the center of mass $\mathbf{V}_{Q}$ :

$$
\mathbf{F}_{f}=-c \mathbf{V}_{Q} \text {. }
$$

This law assumes zero static friction but, is nevertheless reasonable since static friction is quickly overcomed once the egg is set into a rapidly spinning state. The rapidly spinning egg does not transition back to a state of static friction until it stops spinning. This follows from the fact that physically the egg is not in point-contact with the surface; rather, a finite area of the egg is in contact with the surface and is moving relative to the surface as the egg spins. Moreover, it is an experimental fact that eggs rise on oily surfaces. In this case this sliding model of friction is quite reasonable. A more refined model of friction would include rotational torque and dry frictional effects (Coulomb friction).

The slip velocity, i.e., the velocity of the contact point on the rigid body relative to the center of mass, is:

$$
\mathbf{V}_{Q}=\mathbf{V}+\boldsymbol{\omega} \times \mathbf{Q}
$$

where $\mathbf{V}$ is the absolute velocity of the center of mass.

Dimensionless equations of motion. The following dimensionless variables are introduced:

$$
X=b x, \quad Y=b y, \quad Z=b z, \quad T=\frac{t}{\Omega}, \quad \mathbf{F}_{Q}=\frac{I \Omega^{2}}{b} \mathbf{f}_{q}, \quad \mathbf{L}=I \Omega \mathbf{\Upsilon}, \quad \mathbf{Q}=b \mathbf{q}, \quad \mathbf{V}=b \Omega \mathbf{v},
$$

and dimensionless inertia ratio, Froude number, mass, and coefficent of friction respectively:

$$
\sigma=\frac{I_{k}}{I}, \quad \operatorname{Fr}^{-1}=\frac{g}{\Omega^{2} b}, \quad \mu=\frac{M b^{2}}{I}, \quad \nu=\frac{c b^{2}}{I \Omega},
$$

where $\Omega$ is the spin rate of the solution we will linearize about. The dimensionless equations of motion follow,

$$
\begin{aligned}
\mu \ddot{x} & =f_{x}=-\nu\left(\dot{x}+\left(\left(\mathbf{\Upsilon}+\frac{(1-\sigma)}{\sigma}(\mathbf{\Upsilon} \cdot \mathbf{k}) \mathbf{k}\right) \times \mathbf{q}\right) \cdot \mathbf{e}_{x}\right), \\
\mu \ddot{y} & =f_{y}=-\nu\left(\dot{y}+\left(\left(\mathbf{\Upsilon}+\frac{(1-\sigma)}{\sigma}(\mathbf{\Upsilon} \cdot \mathbf{k}) \mathbf{k}\right) \times \mathbf{q}\right) \cdot \mathbf{e}_{y}\right), \\
\mu \ddot{z} & =f_{z}-\mu \mathrm{Fr}^{-1} \\
\dot{\mathbf{\Upsilon}} & =\mathbf{q} \times \mathbf{f}_{q}, \\
\dot{\mathbf{k}} & =\mathbf{\Upsilon} \times \mathbf{k},
\end{aligned}
$$


where $\mathbf{f}_{q}=f_{x} \mathbf{e}_{x}+f_{y} \mathbf{e}_{y}+f_{z} \mathbf{e}_{z}$ and $\mathbf{v}=\dot{x} \mathbf{e}_{x}+\dot{y} \mathbf{e}_{y}+\dot{z} \mathbf{e}_{z}$. The constraint equation expressed in dimensionless form is:

$$
z=-\mathbf{q} \cdot \mathbf{e}_{z} \cdot
$$

Together (8) and (9) are a system of differential algebraic equations. However, one can eliminate the $z$-coordinate using (9) and solve for the normal reaction force $f_{z}$ to obtain a system of differential equations. An explicit expression for the normal reaction force is given in the appendix.

Alternatively, a Lagrangian description of the dynamics starts from the configuration space $Q=\operatorname{SO}(3) \times \mathbb{R}^{2}$. The basic phase space $T Q$ is typically parametrized by rotational and translational coodinates and their conjugate momenta. The angular momentum coordinates that appear in (8) are derived from cotangent bundle reduction on $T Q$. The equations of motion can then be formulated by the Lagrange d'Alembert principle with generalized forces given by the specified sliding friction force. In this setting the normal reaction force is a force of constraint that is an intrinsic part of the Euler-Lagrange operator.

Jellett momentum map. In this section the Jellett momentum map is shown to be an adiabatic invariant of the equations of motion under a fast top approximation, i.e., as $\epsilon \rightarrow 0$ where $\mathrm{Fr}^{-1} \sim O\left(\epsilon^{2}\right)$ and $\nu \sim O(\epsilon)$. Specifically, the Jellett momentum map will be shown to be invariant on the time scale $t / \epsilon$.

The fast top approximation is motivated by the dependence of the dimensionless parameters on the spin rate: holding all other parameters fixed and allowing $\Omega$ to vary, notice from (7) that $\operatorname{Fr}^{-1} \sim 1 / \Omega^{2}$ and $\nu \sim 1 / \Omega$. As an aid to understanding, the dimensionless quantity $\epsilon$ can be thought of as related to the dimensional quantity $1 / \Omega$.

The dimensionless Jellett momentum map is defined as the angular momentum projected on the contact vector:

$$
\beth=\Upsilon \cdot \mathbf{q}
$$

In the Appendix a proof is provided for why this momentum is not precisely conserved in this setting. In fact, it is shown that conservation of the Jellett momentum map implies that the most general form of the axisymmetric body is a sphere with displaced center of mass.

First, (8) is cast in a form which reveals the terms in (8) in the plane defined by $\mathbf{k}$ and $\mathbf{e}_{z}$ and orthogonal to this plane. For $|n| \neq 1$, the angular momentum can be written as:

$$
\mathbf{\Upsilon}=A \mathbf{k}+B \mathbf{e}_{z}+C \mathbf{k} \times \mathbf{e}_{z}
$$

where

$$
A=\frac{P-n Q}{1-n^{2}}, \quad B=\frac{Q-P n}{1-n^{2}}, \quad C=\frac{\dot{n}}{1-n^{2}}, \quad P=(\mathbf{\Upsilon} \cdot \mathbf{k}), \quad Q=\left(\mathbf{\Upsilon} \cdot \mathbf{e}_{z}\right)
$$

Substituting (11) into (8), ignoring translational effects (when friction is small, translational effects are negligible), and simplifying one obtains,

$$
\begin{aligned}
\dot{A} \mathbf{k}+\dot{B} \mathbf{e}_{z}+A \dot{\mathbf{k}}+\dot{C} \mathbf{k} \times \mathbf{e}_{z}+C \dot{\mathbf{k}} \times \mathbf{e}_{z}= & \left(\frac{\left(\mu \ddot{z}+\mu \mathrm{Fr}^{-1}\right)\left(1-\alpha^{2}\right) n}{\sqrt{\alpha^{2}+\left(1-\alpha^{2}\right) n^{2}}}-\nu C \frac{\alpha^{4}\left(1-n^{2}\right)+n^{2}}{\alpha^{2}+\left(1-\alpha^{2}\right) n^{2}}\right)\left(\mathbf{k} \times \mathbf{e}_{z}\right) \\
& -\nu \frac{\left(-A \alpha^{2}+B\left(1-\alpha^{2}\right) n-\alpha^{2} \frac{(1-\sigma)}{\sigma} P\right)}{\sqrt{\alpha^{2}+\left(1-\alpha^{2}\right) n^{2}}} \mathbf{q} \times\left(\mathbf{k} \times \mathbf{e}_{z}\right) \\
\dot{\mathbf{k}} & =-B\left(\mathbf{k} \times \mathbf{e}_{z}\right)+C \mathbf{e}_{z}-n C \mathbf{k}
\end{aligned}
$$

where $n=\mathbf{k} \cdot \mathbf{e}_{z}$. See Sect. 4 for a more thorough justification for ignoring translational effects. Eliminating $\dot{\mathbf{k}}$ from (12) by substitution and collecting coefficients yields,

$$
\begin{aligned}
& (\dot{A}+B C-n A C) \mathbf{k}+(\dot{B}+A C-n B C) \mathbf{e}_{z} \\
& +\left(\dot{C}-n C^{2}-A B-\frac{\left(\mu \ddot{z}+\mu \mathrm{Fr}^{-1}\right)\left(1-\alpha^{2}\right) n}{\sqrt{\alpha^{2}+\left(1-\alpha^{2}\right) n^{2}}}+\nu C \frac{\alpha^{4}\left(1-n^{2}\right)+n^{2}}{\alpha^{2}+\left(1-\alpha^{2}\right) n^{2}}\right) \mathbf{k} \times \mathbf{e}_{z} \\
& +\nu \frac{\left(-A \alpha^{2}+B\left(1-\alpha^{2}\right) n-\alpha^{2} \frac{(1-\sigma)}{\sigma} P\right)}{\sqrt{\alpha^{2}+\left(1-\alpha^{2}\right) n^{2}}} \mathbf{q} \times\left(\mathbf{k} \times \mathbf{e}_{z}\right)=0 .
\end{aligned}
$$


Taking the dot product of (13) with $\left(\mathbf{k} \times \mathbf{e}_{z}\right)$, the following equation is obtained,

$$
\left(1-n^{2}\right)\left(\dot{C}-n C^{2}-\frac{\mu\left(\ddot{z}+\operatorname{Fr}^{-1}\right)\left(1-\alpha^{2}\right) n}{\sqrt{\alpha^{2}+\left(1-\alpha^{2}\right) n^{2}}}-A B+\nu C \frac{\alpha^{4}\left(1-n^{2}\right)+n^{2}}{\alpha^{2}+\left(1-\alpha^{2}\right) n^{2}}\right)=0 .
$$

Consider (14) with fast spin, $\epsilon \ll 1$, and assume $\dot{n} \sim O(\epsilon)$. This assumption is reasonable since for small friction the spheroid rises slowly and very similar to the one Moffatt et al. make in their commentary. Under the fast top approximation,

$$
C \sim O(\epsilon), \quad \operatorname{Fr}^{-1} \sim O\left(\epsilon^{2}\right), \quad \nu \sim O(\epsilon), \quad \ddot{z} \sim O\left(\epsilon^{2}\right), \quad \dot{C} \sim O\left(\epsilon^{2}\right)
$$

and therefore, (14) reduces to,

$$
A B\left(1-n^{2}\right)=0+O\left(\epsilon^{2}\right)
$$

since all neglected terms are $O\left(\epsilon^{2}\right)$. Moreover,

$$
A\left(1-n^{2}\right)=0+O\left(\epsilon^{2}\right)
$$

since $B \neq 0$. This result has a very nice physical interpretation: the external torques on the body primarily act in the plane defined by the axis of symmetry and the vertical. Thus, neglecting the moments orthogonal to this plane can lead to at most an $O\left(\epsilon^{2}\right)$ error. The fact that $A \sim O\left(\epsilon^{2}\right)$ implies that to $O\left(\epsilon^{3}\right)$ the orbital derivative of the Jellett momentum map vanishes on the time scale $O(t / \epsilon)$. From (8) the orbital derivative of $\boldsymbol{\Upsilon} \cdot \mathbf{q}$ is given as:

$$
\begin{aligned}
(\mathbf{\Upsilon} \cdot \mathbf{q})_{t} & =\mathbf{\Upsilon} \cdot(\mathbf{q})_{t}+(\mathbf{\Upsilon})_{t} \cdot \mathbf{q} \\
& =\mathbf{\Upsilon} \cdot(\mathbf{q})_{t} \quad \text { since }(\mathbf{\Upsilon})_{t} \cdot \mathbf{q}=0(\text { cf. }(8)) \\
& =\left(\alpha^{2}+\left(1-\alpha^{2}\right) n^{2}\right)^{-3 / 2} \dot{n}\left(1-\alpha^{2}\right) \alpha^{2}\left[n \Upsilon_{z}-\mathbf{\Upsilon} \cdot \mathbf{k}\right] \\
& =\left(\alpha^{2}+\left(1-\alpha^{2}\right) n^{2}\right)^{-3 / 2} \dot{n}\left(1-\alpha^{2}\right) \alpha^{2} A\left(1-n^{2}\right) \\
& \sim O\left(\epsilon^{3}\right) .
\end{aligned}
$$

Thus, integrating (15) to a time scale $t / \epsilon$, the integral of the orbital derivative of the Jellett momentum map vanishes with $O\left(\epsilon^{2}\right)$ error which implies that on the time scale $t / \epsilon$ the Jellett momentum map is constant to $O\left(\epsilon^{2}\right)$. Numerical evidence Opus Deiis provided in Table 1 and Fig. 4 to confirm this claim. It is shown that the relative error in assuming the Jellett momentum map is nearly constant is monotonically decreasing in time and $O\left(\epsilon^{2}\right)$.

This result is consistent with the following observation: in the inviscid case $n$ is oscillatory. In the presence of friction, as the spheroid rises these oscillations in $n$ die out. Then the spheroid sweeps through a sequence of states which appear steady in a rotating frame. Thus, at any instant of time, the angular momentum appears to point purely in the vertical direction as the asymptotics predicts, i.e., a direct calculation shows that this statement is equivalent to $A=0$. Moreover, a similar

\begin{tabular}{|c|c|c|c|c|}
\hline$\epsilon$ & $\delta \Upsilon \cdot \mathbf{q}$ & $\delta \Upsilon_{z}$ & $\delta \Upsilon_{k}$ & $\delta \frac{\mathbf{\Upsilon} \cdot \mathbf{q}}{\|\mathbf{q}\|}$ \\
\hline $5.0 \mathrm{e}-01$ & $5.9438 \mathrm{e}-02$ & $3.6430 \mathrm{e}-01$ & $6.2573 \mathrm{e}+01$ & $3.6420 \mathrm{e}-01$ \\
\hline $2.5 \mathrm{e}-01$ & $2.1000 \mathrm{e}-02$ & $3.8737 \mathrm{e}-01$ & $6.0267 e+01$ & $3.8727 \mathrm{e}-01$ \\
\hline $1.25 \mathrm{e}-01$ & $6.0074 \mathrm{e}-03$ & $3.9636 \mathrm{e}-01$ & $5.9367 \mathrm{e}+01$ & $3.9627 \mathrm{e}-01$ \\
\hline $6.25 \mathrm{e}-02$ & $1.5589 \mathrm{e}-03$ & $3.9903 \mathrm{e}-01$ & $5.9100 \mathrm{e}+01$ & $3.9894 \mathrm{e}-01$ \\
\hline $3.125 \mathrm{e}-02$ & $3.9344 \mathrm{e}-04$ & $3.9973 \mathrm{e}-01$ & $5.9030 \mathrm{e}+01$ & $3.9963 \mathrm{e}-01$ \\
\hline $1.5625 \mathrm{e}-02$ & $9.8595 \mathrm{e}-05$ & $3.9991 \mathrm{e}-01$ & $5.9012 \mathrm{e}+01$ & $3.9981 \mathrm{e}-01$ \\
\hline
\end{tabular}
asymptotic result can be derived under the assumption that $n$ is oscillatory with slowly varying period, since the average of the terms in (14) and (15) over a cycle of $n$ is zero.

Table 1 Relative error in assuming Jellett momentum map and other momenta are nearly constant for parameters valued at $\alpha=0.6, \sigma=0.8$, and $\mu=1$. Specifically, $\delta x=\left(x_{T}-x_{0}\right) / x_{0}$, where $x_{T}$ and $x_{0}$ are initial and final values of $x$ and $T$ satisfies: $\epsilon T=800$. Observe that as $\epsilon \rightarrow 0$ the error in the Jellett momentum map decreases quadratically unlike the error in the other quantities.

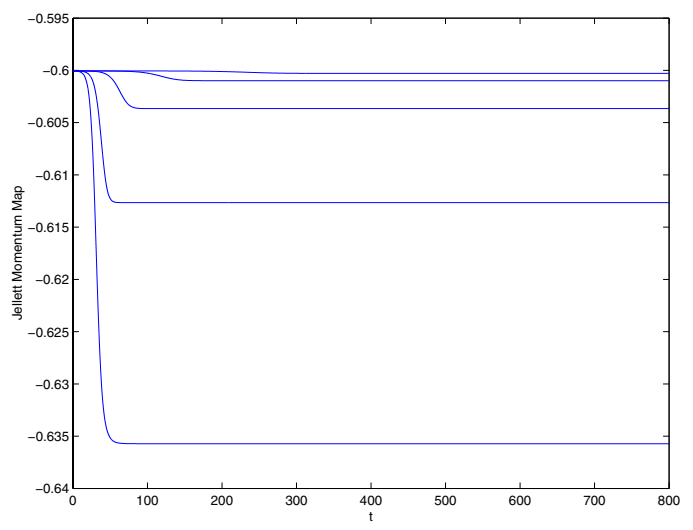

Fig. 4 Plots of the Jellett momentum map for parameters valued at $\alpha=0.6, \sigma=0.8$, and $\mu=1$ and for $\epsilon=0.5,0.25,0.125,0.0625,0.03125$. 


\section{Linear theory applied to non-risen state}

Straightforward application of linear theory determines the spectral stability of the non-risen state of the spheroid (cf. Fig. 1). Since this periodic solution is explicitly known, a simple change to rotating coordinates transforms the non-risen state into a fixed point of the transformed equations of motion.

Transformation to a fixed point. Here the stability of the spinning, non-risen state given by:

$$
\mathbf{\Upsilon}=\mathbf{e}_{z}, \quad \mathbf{k}=(\cos (t), \sin (t), 0), \quad x=y=\dot{x}=\dot{y}=\dot{z}=0, \quad z=\alpha
$$

will be investigated. To analyze the stability of this relative equilibrium, the equation is written in coordinates rotating about the $\mathbf{e}_{z}$ axis with unit angular velocity. This change of coordinates will transform (16) from a periodic solution to a fixed point of (8). To carry out this change of coordinates, the following rotation matrix is defined:

$$
\mathbf{R}_{z}(\theta)=\left[\begin{array}{ccc}
\cos (\theta) & \sin (\theta) & 0 \\
-\sin (\theta) & \cos (\theta) & 0 \\
0 & 0 & 1
\end{array}\right]
$$

In terms of this matrix the transformed coordinates are:

$$
\mathbf{v}^{\prime}=\mathbf{R}_{z}(t) \mathbf{v}, \quad \mathbf{k}^{\prime}=\mathbf{R}_{z}(t) \mathbf{k}, \quad \mathbf{\Upsilon}^{\prime}=\mathbf{R}_{z}(t) \mathbf{\Upsilon}
$$

In terms of these rotating variables the governing equations become (where 's are eliminated to simplify expressions):

$$
\begin{aligned}
\mu \ddot{x} & =f_{x}+\mu \dot{y}, \\
\mu \ddot{y} & =f_{y}-\mu \dot{x}, \\
\mu \ddot{z} & =f_{z}-\mu \mathrm{Fr}^{-1}, \\
\dot{\mathbf{\Upsilon}} & =\mathbf{q} \times \mathbf{f}_{q}+\mathbf{\Upsilon} \times \mathbf{e}_{z}, \\
\dot{\mathbf{k}} & =\mathbf{\Upsilon} \times \mathbf{k}+\mathbf{k} \times \mathbf{e}_{z} .
\end{aligned}
$$

In rotating coordinates (16) becomes,

$$
\Upsilon=\mathbf{e}_{z}, \quad \mathbf{k}=\mathbf{e}_{x}, \quad x=y=\dot{x}=\dot{y}=\dot{z}=0, \quad z=\alpha,
$$

which is a fixed point of (17). Linearizing (17) about this equilibrium yields,

$$
\begin{aligned}
\mu \ddot{x} & =-\nu \dot{x}+\mu \dot{y}+\alpha \nu \sigma \Upsilon_{y}, \\
\mu \ddot{y} & =-\nu \dot{y}-\mu \dot{x}-\alpha \nu \sigma \Upsilon_{x}+\nu \frac{\sigma-\alpha^{2}}{\alpha \mu \sigma}, \\
\dot{\Upsilon}_{x} & =-\alpha \nu \dot{y}-\frac{\alpha^{2} \nu}{\sigma} \Upsilon_{x}+\Upsilon_{y}+\left(\nu-\frac{\alpha^{2} \nu}{\sigma}\right) n, \\
\dot{\Upsilon}_{y} & =\alpha \nu \dot{x}-\Upsilon_{x}-\alpha^{2} \nu \Upsilon_{y}+\frac{\operatorname{Fr}^{-1}\left(1-\alpha^{2}\right) \mu}{\alpha} n, \\
\dot{m} & =\Upsilon_{z}, \\
\dot{n} & =-\Upsilon_{y},
\end{aligned}
$$

where $\Upsilon=\Upsilon_{x} \mathbf{e}_{x}+\Upsilon_{y} \mathbf{e}_{y}+\Upsilon_{z} \mathbf{e}_{z}$ and $\mathbf{k}=l \mathbf{e}_{x}+m \mathbf{e}_{y}+n \mathbf{e}_{z}$.

Reduction by translational symmetry. Suppose the translation of the center of mass is negligible. This assumption is rigorously true for small friction. In the inviscid case, (8) shows that for an initially uniformly rotating state the center of mass does not move at all. An application of perturbation theory for small friction, i.e., $\nu \ll 1$, reveals this result holds to leading order in $\nu$. 
Moreover, this assumption is fully borne out in simulation and numerical stability analysis of the full system. See BouRabee et al. [4] for a very similar and more complete justification of why ignoring translational effects in tippe top inversion is a reasonable assumption. Thus, (18) reduces to:

$$
\begin{aligned}
\dot{\Upsilon}_{x} & =-\frac{\alpha^{2} \nu}{\sigma} \Upsilon_{x}+\Upsilon_{y}+\left(\nu-\frac{\alpha^{2} \nu}{\sigma}\right) n, \\
\dot{\Upsilon}_{y} & =-\Upsilon_{x}-\alpha^{2} \nu \Upsilon_{y}+\frac{\operatorname{Fr}^{-1}\left(1-\alpha^{2}\right) \mu}{\alpha} n, \\
\dot{m} & =\Upsilon_{z}, \\
\dot{n} & =-\Upsilon_{y},
\end{aligned}
$$

which can be rewritten as a cubic polynomial in the nutation angle $n$,

$$
\dddot{n}+\alpha^{2} \nu \frac{1+\sigma}{\sigma} \ddot{n}+\left(1+\frac{\alpha^{4} \nu^{2}}{\sigma}+\mu \operatorname{Fr}^{-1} \frac{\left(1-\alpha^{2}\right)}{\alpha}\right) \dot{n}+\nu \frac{\operatorname{Fr}^{-1} \alpha\left(1-\alpha^{2}\right) \mu+\left(\alpha^{2}-\sigma\right)}{\sigma} n=0 .
$$

The stability of (20) is determined by the roots of a cubic polynomial of the form:

$$
\lambda^{3}+a_{2} \lambda^{2}+a_{3} \lambda+a_{4}=0
$$

By the Routh-Hurwitz criterion (see, eg, Gantmacher [6]), the necessary and sufficient conditions for this cubic polynomial to be stable (spectrum in left-half plane) are:

$$
\begin{array}{r}
h_{1}=\alpha^{2} \nu \frac{(1+\sigma)}{\sigma}>0, \\
h_{2}=\nu \frac{\left(\operatorname{Fr}^{-1} \alpha\left(1-\alpha^{2}\right) \mu+\left(\alpha^{2}-\sigma\right)\right)}{\sigma}>0, \\
h_{3}=\nu\left(\operatorname{Fr}^{-1} \alpha\left(1-\alpha^{2}\right) \mu+1+\alpha^{2}+\frac{\alpha^{6} \nu^{2}(1+\sigma)}{\sigma^{2}}\right)>0 .
\end{array}
$$

Non-Risen state stability analysis. Numerical stability results from the complete system (18) reveal that these criteria correctly predict the stability of the non-risen state. Moreover, for parameter values that have physical meaning (positive friction, inertia ratio, etc.), $h_{2}$ is the only term which could ever become negative.

Figs. 5, 6, and 7 show plots of $h_{2}$ as a function of $\alpha$ in the parameter space defined by $\mathrm{Fr}^{-1}, \sigma$ and $\nu$. Fig. 5 shows that increasing $\mathrm{Fr}^{-1}$ can stabilize the non-risen state. As the $\mathrm{Fr}^{-1}$ increases, the gravitational force increases compared to the forces due to spin. Thus, this result makes sense since the gravitational force acts against the forces due to spin which

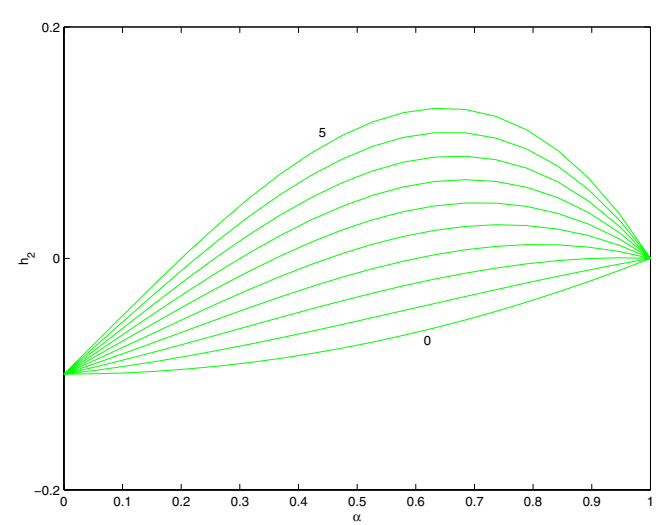

Fig. 5 Here $h_{2}$ is plotted as a function of $\alpha$ for varying Froude number, $0 \leq \operatorname{Fr}^{-1} \leq 5$. The stability of the non-risen state $n=$ $0, \Upsilon_{z}=1$ is determined by $h_{2}>0$ (cf. (21)). Parameters are valued at $\nu=0.1, \sigma=1, \mu=1$.

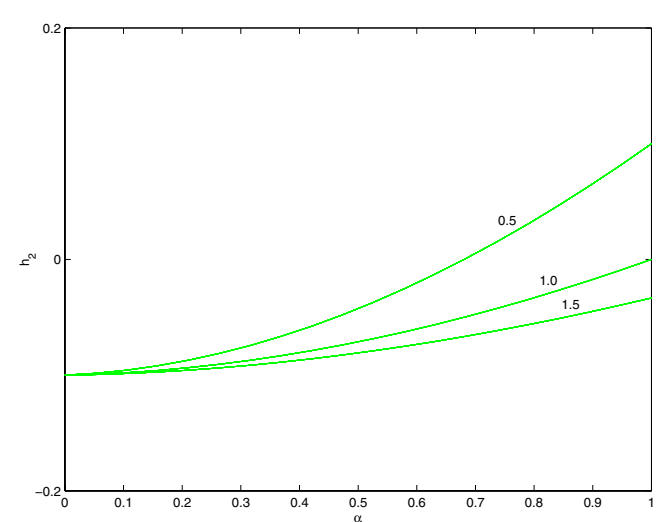

Fig. 6 Here $h_{2}$ is plotted as a function of $\alpha$ for varying inertia ratio, $\sigma$. The stability of the non-risen state $n=0, \Upsilon_{z}=1$ is determined by $h_{2}>0$ (cf. (21)). Parameters are valued at $\nu=$ $0.1, \mathrm{Fr}^{-1}=0.1, \mu=1$. 


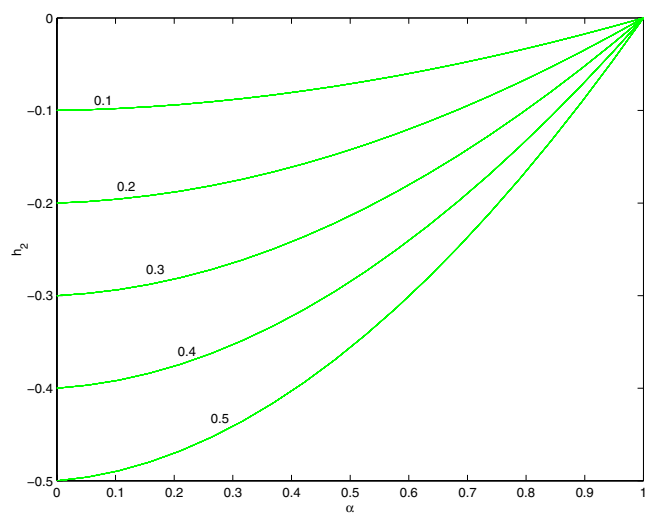

Fig. 7 Here $h_{2}$ is plotted as a function of $\alpha$ for varying dimensionless friction, $\nu$. The stability of the risen state $n=0, \Upsilon_{z}=1$ is determined by $h_{2}>0$ (cf. (21)). Parameters are valued at $\nu=0.1, \sigma=1, \mathrm{Fr}^{-1}=0.1$

induce the spheroid to rise. However, for parameter values used in the simulations in this paper, the non-risen state is always unstable, i.e., $\operatorname{Fr}^{-1}=0.1,0.2,0.3$.

As the inertia ratio decreases the rotational inertia about the equatorial axis increases compared to the rotational inertia about the axis of symmetry. The non-risen state becomes unstable only if the rotational inertia about the equatorial axis is overcomed. Fig. 6 confirms this fact, i.e., decreasing the inertia ratio can stabilize the non-risen state by increasing the rotational moment of inertia about the equatorial axes of the spheroid. An instructive way to understand this limit is to put a heavy rod through the center of the spheroid and allow its radius to shrink holding all other parameters fixed. Clearly in this limit, the spheroid will not want to rise and tend to spin with the rod horizontal.

Finally, the magnitude of nonzero dissipation has no effect on the stability of the non-risen state as shown in Fig. 7.

Normal form for non-risen state. Here (19) will be examined more closely. For clarity's sake, it helps to view the equations in dimensional form; (19) in dimensional form becomes:

Standard Damping term Gyroscopic term

Rotational Damping term

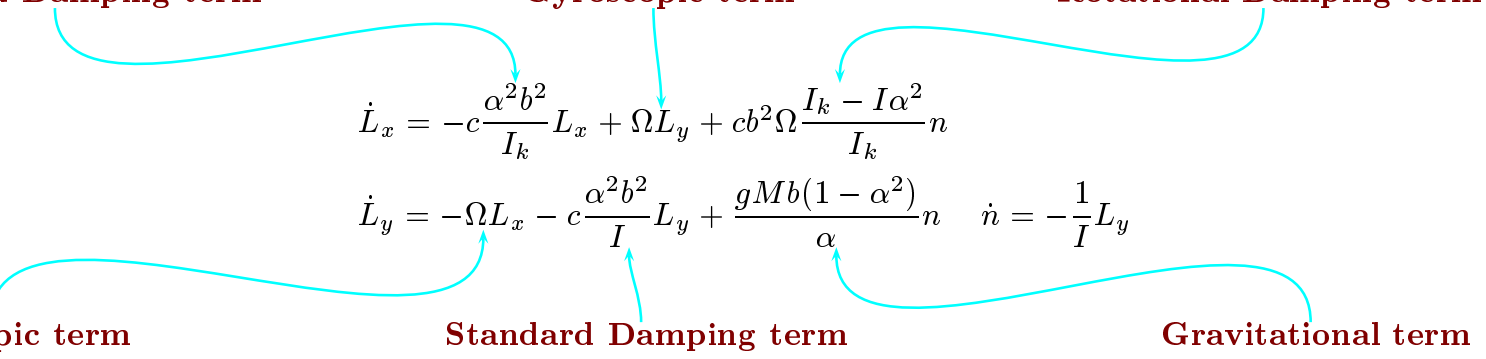

\section{Gyroscopic term}

Standard Damping term

Gravitational term

Since the differential equation involving $m$ in (19) decouples from the other equations, it is not included. Thus, the system reduces to three degrees of freedom. Without friction, $c=0$, the equation becomes a balance between Coriolis and gravitational effects. In particular, when $\Omega=0$, the equations become a simple harmonic oscillator in $n$ with positive spring constant $g M R\left(1-\alpha^{2}\right) / I$. When $\Omega=0$ and $c>0$, the spheroid rocks back and forth with damped oscillations. When $\Omega \neq 0$, the gyroscopic and rotational damping term linearly proportional to $\Omega$ appear in the equations.

In order to understand the effect of each of the four terms on the stability of (22)'s zero solution better, the following parameters are introduced,

$$
\gamma=\frac{g\left(1-\alpha^{2}\right) M b}{\alpha}, \quad \beta=c \alpha^{2} b^{2}, \quad \delta=c b^{2} \Omega \frac{I_{k}-I \alpha^{2}}{I_{k}} .
$$

In terms of these parameters (22) become,

$$
\begin{aligned}
& \dot{L}_{x}=-\frac{\beta}{I_{k}} L_{x}+\Omega L_{y}+\delta n, \\
& \dot{L}_{y}=-\Omega L_{x}-\frac{\beta}{I} L_{y}+\gamma n, \quad \dot{n}=-\frac{1}{I} L_{y} .
\end{aligned}
$$

It is easily verified that the stability criteria for (23) is determined by $h_{2}^{d}=c\left(\beta \gamma-I_{k} \delta \Omega\right) /\left(I I_{k}\right)>0$. From this inequality it is clear that standard damping, $\epsilon$, cannot destabilize the non-risen state and therefore cannot explain egg rising. Similarly, the gravitational term, $\delta$, stabilizes the non-risen state and therefore cannot explain egg rising. Only when $I_{k} \delta \Omega>\beta \gamma$ is the non-risen state unstable. Thus, only rotational damping can induce an instability in the gravitationally stable non-risen state. 
A straightforward application of the perturbation theory of polynomials to (23)'s cubic characteristic polynomial, confirms this result. For $c$ small roots of (23) have the form,

$$
\lambda \sim \lambda_{0}+c \lambda_{1}
$$

where $z_{0}(23)$ satisfies the following unperturbed (frictionless) polynomial:

$$
\lambda_{0}^{3}+\left(\Omega^{2}+\frac{\gamma}{I}\right) \lambda_{0}=0
$$

with solutions given by:

$$
\lambda_{0}=0, \pm i \sqrt{\Omega^{2}+\frac{\gamma}{I}} .
$$

The first-order correction to $\lambda_{0}= \pm i \sqrt{\Omega^{2}+\frac{\gamma}{I}}$ predicts roots with negative real parts. However, the first-order correction to $\lambda_{0}=0$ predicts an instability if $I_{k} \delta \Omega>\beta \gamma$ :

$$
\lambda_{1}=\frac{\left(I_{k} \delta \Omega-\beta \gamma\right)}{I_{k}\left(I \Omega^{2}+\gamma\right)}
$$

which is proportional to $h_{2}^{d}$.

\section{Linear theory applied to risen state}

In this Sect.(8) is linearized about the risen state. The risen state is shown to be completely determined if its angular momentum is known. To determine the asymptotic value of the angular momentum of the risen state from an initially non-risen state, the dynamics is restricted to level curves of the Jellett momentum map, an adiabatic invariant of (8). On this level curve, equilibria are derived and their stability investigated. These equilibria correspond to the risen state. It is shown that the stability of the risen state is fully determined by the modified Maxwell-Bloch equations, see Bou-Rabee et al. [4].

Derivation of equilibria. Equilibria of (8) satisfy:

$$
\begin{aligned}
& \boldsymbol{\Upsilon} \times \mathbf{k}=0 \Longrightarrow \mathbf{\Upsilon} \text { and } \mathbf{k} \text { are collinear }, \\
& \mathbf{e}_{z} \times \mathbf{k}=0 \Longrightarrow \mathbf{e}_{z} \text { and } \mathbf{k} \text { are collinear } .
\end{aligned}
$$

Therefore, equilibria satisfy:

$$
\dot{x}=\dot{y}=x=y=\Upsilon_{x}=\Upsilon_{y}=l=m=0, \quad \Upsilon_{z}=\text { constant }, \quad n= \pm 1 .
$$

For a fixed value of the Jellett momentum map,

$$
\Upsilon \cdot \mathbf{q}=-\alpha
$$

an adiabatic invariant of (8), two equilibria are specifically given by:

$$
\dot{x}=\dot{y}=x=y=\Upsilon_{x}=\Upsilon_{y}=l=m=0, \quad \Upsilon_{z}=\alpha, \quad n= \pm 1 .
$$

These equilibria correspond to the risen state shown in Fig. 1.

Linearization about risen state. Linearizing the equations of motion about these states $\left(n= \pm 1, \Upsilon_{z}=\alpha\right)$ and writing the resulting equations in terms of the complex variables:

$$
V_{C}=\dot{x}+i \dot{y}, \quad \Lambda=\Upsilon_{x}+i \Upsilon_{y}, \quad \Phi=l+i m
$$

one obtains:

$$
\begin{aligned}
\mu \dot{V}_{C} & =-\nu V_{C}-i \nu \Lambda \pm i \nu \frac{\alpha\left(\alpha^{2}-\sigma\right)}{\sigma} \Phi \\
\dot{\Lambda} & =i \nu V_{C}-\nu \Lambda \pm\left(\nu \frac{\alpha\left(-\alpha^{2}+\sigma\right)}{\sigma}+i \operatorname{Fr}^{-1}\left(1-\alpha^{2}\right) \mu\right) \Phi \\
\dot{\Phi} & =\mp i \Lambda+i \alpha \Phi .
\end{aligned}
$$


Assuming a solution of the form $\mathrm{e}^{\lambda t} \phi$ we obtain the following eigenvalue problem:

$$
\lambda \phi=\nu \mathbf{C} \phi+i \mathbf{K} \phi,
$$

where

$$
\mathbf{C}=\left[\begin{array}{ccc}
-\frac{1}{\mu} & -i \frac{1}{\mu} & \pm i \frac{\alpha\left(\alpha^{2}-\sigma\right)}{\sigma \mu} \\
i \nu & -\nu & \pm \frac{\alpha\left(-\alpha^{2}+\sigma\right)}{\sigma} \\
0 & 0 & 0
\end{array}\right], \quad \mathbf{K}=\left[\begin{array}{ccc}
0 & 0 & 0 \\
0 & 0 & \pm i \operatorname{Fr}^{-1}\left(1-\alpha^{2}\right) \mu \\
0 & \mp 1 & \alpha \sigma
\end{array}\right]
$$

Rising egg modified Maxwell Bloch equations. If translational effects are ignored, the linearized equations (25) simplify:

$$
\begin{aligned}
& \dot{\Lambda}=-\nu \Lambda \pm\left(\nu \frac{\alpha\left(-\alpha^{2}+\sigma\right)}{\sigma}+i \operatorname{Fr}^{-1}\left(1-\alpha^{2}\right) \mu\right) \Phi, \\
& \dot{\Phi}=\mp i \Lambda+i \alpha \Phi .
\end{aligned}
$$

This assumption is reasonable for the same reasons provided for reducing the equations linearized about the non-risen state.

Eq. (27) can be rewritten in terms of $\Phi$ alone:

$$
\ddot{\Phi}+i a \dot{\Phi}+b \dot{\Phi}+i c \Phi+d \Phi=0
$$

where:

$$
a=-\alpha, \quad b=\nu, \quad c=-\frac{\alpha^{3} \nu}{\sigma}, \quad d=\operatorname{Fr}^{-1}\left(-1+\alpha^{2}\right) \mu .
$$

(28) are in the form of the modified Maxwell-Bloch equations. It has already been shown that rotational damping is critical to explaining the instability of the gravitationally stable non-risen state. Based on the stability properties of modified MaxwellBloch systems (see Bou-Rabee et al. [4]), the rotational damping term in this setting explains why the gyroscopically stabilized risen state spins stably. In fact, the presence of only standard damping destabilizes the gyroscopically stable risen state. Only when the rotational and standard damping term are in the right ratio, is the risen state stable. Thus, just as in tippe top inversion, the rotational damping term is essential to explain why the gravitationally stable non-risen state becomes unstable and the gyroscopically stable risen state is stable. Hence, the term is essential to the egg rising phenomenon, and in that sense, (27) and (19) are normal forms for the egg rising phenomenon.

Risen state stability analysis. The stability criteria for modified Maxwell-Bloch systems is explicitly given in Proposition 2.2 of Bou-Rabee et al. [4]. The stability criteria for the risen state $\left(n= \pm 1, \Upsilon_{z}^{*}=\alpha\right)$ :

$$
\begin{array}{r}
h_{4}=\nu^{2}\left(\alpha^{4} \frac{\left(\sigma-\alpha^{2}\right)}{\sigma^{2}}-\operatorname{Fr}^{-1}\left(1-\alpha^{2}\right) \mu\right)>0, \\
h_{5}=\nu\left(\alpha^{2} \frac{\left(\sigma-\alpha^{2}\right)}{\sigma}-\operatorname{Fr}^{-1}\left(1-\alpha^{2}\right) \mu+\nu^{2}\right)>0 .
\end{array}
$$

The first inequality consistently dictates stability, since a straightforward calculation shows that

$$
h_{4}>0 \Rightarrow \frac{\sigma}{\alpha^{2}}>1 \Rightarrow \frac{\sigma^{2}}{\alpha^{4}}>\frac{\sigma}{\alpha^{2}} \Rightarrow h_{5}>0 .
$$

Observe $h_{4}$ is independent of the magnitude of the dimensionless friction similar to the non-risen state.

Figs. 8, 9, and 10 show how the stability of the risen state varies in parameter space. Fig. 8 shows that the higher the Froude number the more likely the risen state is stable since in this limit gyroscopic forces will outweigh gravitational ones. Also, Fig. 10 confirms that stability (or lack thereof) of the risen state is independent of the magnitude of the dimensionless friction.

The dependence of $h_{4}$ on the inertia ratio is interesting. For $\sigma \rightarrow 0$, place a heavy rod through the axis of symmetry and let its radius vary. As the radius of the rod decreases holding all other parameters fixed, the moment of inertia about the axis of symmetry decreases relative to the moment of inertia about the equatorial axes. In this limit the spheroid in the non-risen state will tend to not rise as stated earlier, i.e., it will tend to the non-risen state where the rod is horizontal. Similarly, Fig. 9 shows for $\sigma<1$ decreasing the inertia ratio destabilizes the risen state by reducing the gyroscopic forces which stabilize this state. 


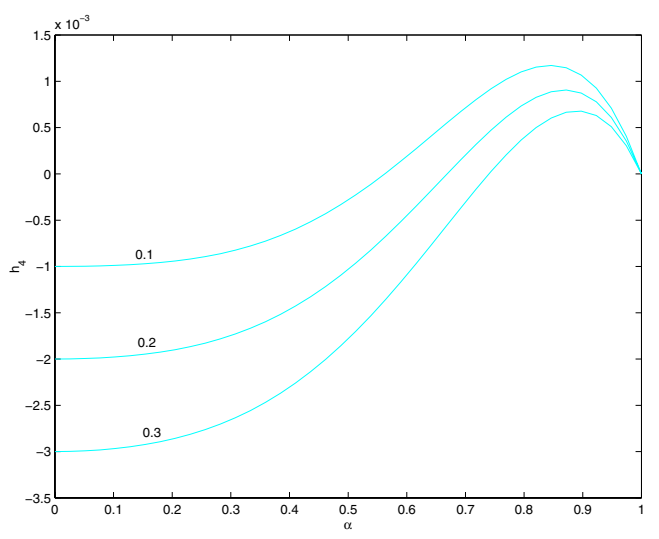

Fig. 8 Here $h_{4}$ is plotted as a function of $\alpha$ for $\mathrm{Fr}^{-1}=$ $0.1,0.2,0.3$ as labelled. The stability of the risen state $n=$ $1, \Upsilon_{z}^{*}=\alpha$ is determined by $h_{4}>0$ (cf. (29)). Parameters are valued at $\nu=0.1, \sigma=1, \mu=1$.

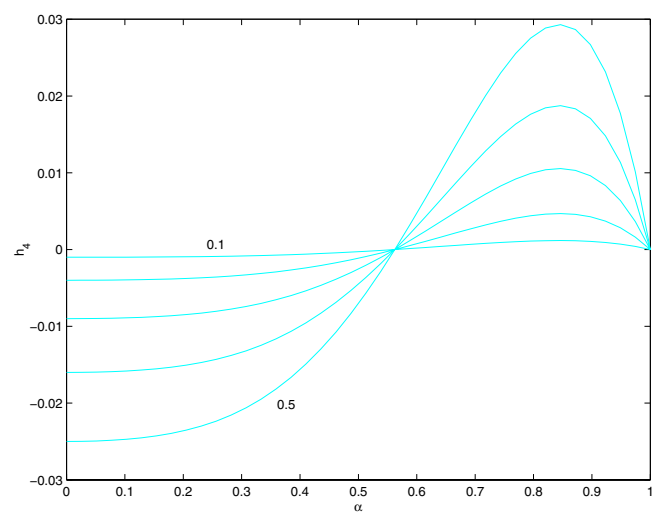

Fig. 10 Here $h_{4}$ is plotted as a function of $\alpha$ for varying dimensionless friction, $\nu$. The stability of the risen state $n=1, \Upsilon_{z}^{*}=\alpha$ is determined by $h_{4}>0$ (cf. (29)). Parameters are valued at $\mathrm{Fr}^{-1}=0.1, \mu=1, \sigma=1$.

For the special case $\alpha=1$, the spheroid becomes a sphere with center of mass at its geometric center is always in a gravitationally favorable position. As $\sigma$ becomes small, the sphere will tend to a state where it spins with the rod horizontal.

To understand $\sigma>1$, place a ring at the equator of the spheroid. Clearly, the risen state in such a setting can become unstable since destabilizing gravitational effects can exceed the gyroscopic forces that stabilize the spheroid. In addition, assuming the Jellett momentum map is constant and the geometry of the spheroid remains fixed, the kinetic energy of the asymptotic risen state becomes small as $\sigma$ becomes large, since $E_{\text {risen }}=\alpha^{2} / \sigma$. However, the momentum must remain constant if the Jellett momentum is assumed to stay nearly fixed. These two facts imply that the angular velocity of the risen state decreases, and hence, the gyroscopic and rotational damping forces which stabilize the gravitationally unstable spheroid decrease. But, even if $\sigma$ is large, in the limit as $\alpha \rightarrow 1$ gravitational effects become less important since the spheroid tends to an energetically favorable state. These trends are observed in Fig. 9 for $\sigma>1$.

For the special case $\alpha=1$, the spheroid becomes a sphere with center of mass at its geometric center is always in a gravitationally favorable position. If $\sigma$ is large, the sphere will tend to a state where it spins about the perpendicular axis of the ring.

Risen state stable $\rightarrow$ non-risen state unstable. A very interesting result is proven now: if the risen state is stable then the non-risen state is unstable. Suppose the risen state is stable. Then from (29),

$$
h_{4}>0 \Rightarrow\left(\sigma-\alpha^{2}\right)>\frac{\sigma^{2} \operatorname{Fr}^{-1}\left(1-\alpha^{2}\right) \mu}{\alpha^{4}} .
$$

From (21), the non-risen state is unstable if and only if,

$$
h_{2}<0 \Rightarrow\left(\sigma-\alpha^{2}\right)>\alpha \operatorname{Fr}^{-1}\left(1-\alpha^{2}\right) \mu \text {. }
$$

Thus, the risen state is stable implies the non-risen state is unstable if,

$$
\frac{\sigma^{2}}{\alpha^{4}} \geq \alpha \Rightarrow \sigma^{2} \geq \alpha^{5} \text {. }
$$


However, the only way the non-risen state can be stable is if $\sigma>\alpha^{2}$ which implies $\sigma^{2}>\alpha^{4}>\alpha^{5}$ since $0<\alpha \leq 1$. Thus, when the risen state is stable the non-risen state is unstable.

\section{Nonlinear theory}

The nonlinear theory is made possible by assuming that the Jellett momentum map is nearly constant over the slow time scale, i.e., is an adiabatic invariant of the equations of motion. This assumption simplifies the dynamics in phase space to level curves of the Jellett momentum map. In this section the energy landscape on these level curves is investigated and a global connection between the non-risen and risen states established.

Egg asymptotic states. To establish the existence of a orbit connecting the asymptotically stable and unstable states of the theoretical egg, LaSalle's invariance principle will be used (cf. Theorem 2.3.4 Bloch [1]). Consider a vector field $\chi$ on a manifold $P$. Let $V$ be a Lyapunov function with negative semi-definite orbital derivative: $V_{t} \leq 0$ for all $z \in P$. We define the set $\aleph:=\left\{z \in P \mid V_{t}(z)=0\right\}$.

Theorem 5.1 (LaSalle's principle) Let $z:[0, \infty) \rightarrow P$, be an integral curve of a vector field $\chi$ with initial condition $z(0)=z_{0}$. Suppose there is a positively invariant set (trapping region) $M$ such that $z(t) \in M$ for all $t \geq 0$. Then $z(t)$ converges to the largest subset of $\aleph \cap M$ that is invariant under the flow of $\chi$ for all $t$, positive and negative.

For configuration variables which satisfy the constraint (9), the energy of the viscous spheroid is:

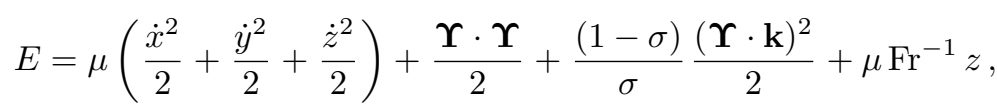

which is a sum of translational, rotational, and gravitational effects. The energy's orbital derivative is given by:

$$
E_{t}=-\nu\left\|\mathbf{v}_{Q}\right\|^{2}
$$

where $\left\|\mathbf{v}_{Q}\right\|$ is the norm of the dimensionless slip velocity (cf. eq. (6)). The energy's orbital derivative is negative semidefinite which implies that the energy decreases monotonically until the slip velocity vanishes.

No slip and no force problem. We now mention some properties of the asymptotic state where the slip velocity vanishes and energy is conserved. (8), the governing equations, without slip reduce to the system:

$$
\begin{aligned}
\ddot{x} & =0 \\
\ddot{y} & =0 \\
\dot{\boldsymbol{\Upsilon}} & =\frac{\left(1-\alpha^{2}\right) f_{z} n}{\sqrt{\alpha^{2}+\left(1-\alpha^{2}\right) n^{2}}}\left(\mathbf{e}_{z} \times \mathbf{k}\right), \\
\dot{\mathbf{k}} & =\sigma \mathbf{\Upsilon} \times \mathbf{k}
\end{aligned}
$$

where there is no tangential surface force. Clearly, this system is Hamiltonian and in particular, it conserves energy. A lengthy calculation gives,

$$
\mathbf{v}_{Q}=0 \Longrightarrow \dot{n}=0 \Longrightarrow \dot{\Upsilon}_{z}=0 \Longrightarrow \Upsilon \cdot\left(\mathbf{e}_{z} \times \mathbf{k}\right)=0 \Longrightarrow \dot{x}=\dot{y}=0
$$

Solutions are therefore defined by level sets of $l^{2}+m^{2}$ and $\left(\Upsilon_{x}\right)^{2}+\left(\Upsilon_{y}\right)^{2}$. For $n \neq \pm 1$ these solutions are described by precession, spin about the axis of symmetry, and no nutation. These are similar to the tumbling solutions observed in the tippe top, see Bou-Rabee et al. [4].

The abundance of invariant quantities in the no slip, no force problem severely limits the behavior of solutions to eq. (32). In fact, the analysis above reveals that the angular momentum about the vertical and the nutation angle fully determine solutions of (32). The nutation angle $\theta$ is the angle the axis of symmetry makes with the vertical $\mathbf{e}_{z}$ :

$$
n=\cos (\theta) .
$$

Therefore, it is reasonable to suppose that the initially sliding, viscous egg will tend to a Lagrange top solution where $\Upsilon_{z}$ and $n$ extremize the energy on the approximately invariant manifold defined by a fixed value of the Jellett momentum map. In terms of LaSalle's principle, $\aleph$ is the set of all energy-conserving asymptotic solutions defined by $\Upsilon_{z}$ and $n$ which extremize the energy subject to the adiabatic constraint that $\mathbf{\Upsilon} \cdot \mathbf{q}=-\alpha$. 
Energy-adiabatic momentum minimization. Minima of the total energy subject to the adiabatic angular momentum constraint, $\varphi_{J}=\mathbf{\Upsilon} \cdot \mathbf{q}+\alpha=0$ (cf. (10)), the attitude constraint, $\varphi_{k}=\mathbf{k} \cdot \mathbf{k}-1=0$, and the surface constrain $\varphi_{z}=z+\mathbf{q} \cdot \mathbf{e}_{z}=0$ are derived here. The augmented energy is given by,

$$
h=E+\lambda_{J} \varphi_{J}+\lambda_{k} \varphi_{k}+\lambda_{z} \varphi_{z}
$$

where $\lambda_{J, k, z}$ are Lagrange multipliers. Extrema of $h$ satisfy:

$$
\dot{x}=\dot{y}=\dot{z}=0
$$

which indicate that the center of mass and nutation angle remain fixed. The angular momentum in terms of $n$ takes the form:

$$
\Upsilon=\frac{\lambda_{J}}{z}\left(n\left(\sigma-\alpha^{2}\right) \mathbf{k}+\alpha^{2} \mathbf{e}_{z}\right)
$$

And the constraint $\varphi_{J}=0$ implies,

$$
\lambda_{J}=\frac{\alpha\left(\alpha^{2}-n^{2}\left(-1+\alpha^{2}\right)\right)}{\left(1-n^{2}\right) \alpha^{4}+n^{2} \sigma} .
$$

With the angular momentum in the above form, the slip velocity vanishes: $\mathbf{v}_{Q}=0$. This result is expected since extrema of the energy satisfy the no-slip, no-force problem. Moreover, extrema of the energy now satisfy:

$$
\begin{aligned}
& \frac{-2 \alpha^{2} \lambda_{k}+n^{2}\left(\alpha^{4} \lambda_{J}^{2}-2 \lambda_{k}+\sigma^{2} \lambda_{J}^{2}+2 \alpha^{2}\left(\lambda_{k}-\lambda_{J}^{2} \sigma\right)\right)}{\left.-\alpha^{2}+n^{2}\left(-1+\alpha^{2}\right)\right) \sigma} \mathbf{k} \\
& +n \frac{\alpha^{2} \lambda_{J}^{2}\left(\alpha^{2}-\sigma\right)+\left(-1+\alpha^{2}\right)\left(\alpha^{2}-n^{2}\left(-1+\alpha^{2}\right)\right)^{3 / 2} \lambda_{z}}{\left(\alpha^{2}-n^{2}\left(-1+\alpha^{2}\right)\right)^{2}} \mathbf{e}_{z}=0 .
\end{aligned}
$$

When $\mathbf{e}_{z}$ and $\mathbf{k}$ are linearly dependent, the minimizer is the risen state given by $n= \pm 1$ and $\mathbf{\Upsilon}=\alpha \mathbf{e}_{z}$. When the two vectors are linearly independent, $\lambda_{k}$ is chosen so that the coefficient of $\mathbf{k}$ vanishes. When $n=0$ the minimizer is the non-risen state given by $\mathbf{\Upsilon}=\mathbf{e}_{z}$. So far, the risen and non-risen states are the only minimizers of the constrained energy. The remaining minimizers satisfy the following simplified equation derived from the coefficient of $\mathbf{e}_{z}$,

$$
\frac{\operatorname{Fr}^{-1}\left(1-\alpha^{2}\right) \mu}{\sqrt{\alpha^{2}+\left(1-\alpha^{2}\right) n^{2}}}+\frac{\alpha^{4}\left(\alpha^{2}-\sigma\right)}{\left(\left(-1+n^{2}\right) \alpha^{4}-n^{2} \sigma\right)^{2}}=0 .
$$

Criteria for egg rising. The explicit form of the solutions of (33) are not necessary to derive criteria for the rising phenomenon. Rather, the location of the roots of this equation in the complex plane will suffice since if $|n|>1$ the extrema violate the constraint $\varphi_{k}=\mathbf{k} \cdot \mathbf{k}-1=0$ and if there exists extrema with $|n| \leq 1 \neq 0$ starting at $n=0$ the asymptotic state will not be $n=1$, implying $n=1$ is asymptotically unstable. Thus, the problem of finding all eligible extrema of energy is converted into determining the stability of a polynomial. To accomplish this analysis, (33) is transformed to z-coodinates via,

$$
z=\sqrt{\alpha^{2}+\left(1-\alpha^{2}\right) n^{2}}
$$

to obtain a quartic polynomial in $z$. In these coordinates the requirement that $|n| \leq 1$ becomes

$$
1 \geq z \geq \alpha^{2}
$$

To determine whether all roots satisfy this criterion the following transformation in the complex domain is used:

$$
w=\frac{-1+z}{z-\alpha^{2}}
$$

which describes a mapping from the region outside the open disc $\left|z-\frac{1+\alpha^{2}}{2}\right| \leq \frac{1-\alpha^{2}}{2}$ to the open left half plane in $w$. In terms of $w$ the quartic polynomial reads:

$$
a_{0} w^{4}+a_{1} w^{3}+a_{2} w^{2}+a_{3} w+a_{4}=0 .
$$

Specific values of these coefficients are provided in the appendix.

The stability of the polynomial in $w$ determines if any roots are physical, i.e., if it is stable then there are no other physical extrema. The Liénard and Chipart criterion applied to quartic polynomials determines it's stability (cf. Gantmacher [6]). 


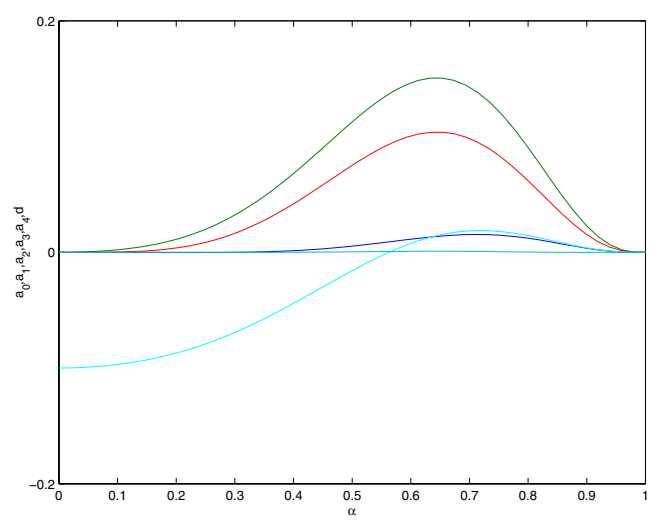

Fig. 11 Plot of the coefficients of the quartic in w (34) for $\mu=$ $1.0, \mathrm{Fr}^{-1}=0.1, \sigma=1.0$, and variable $\alpha$.

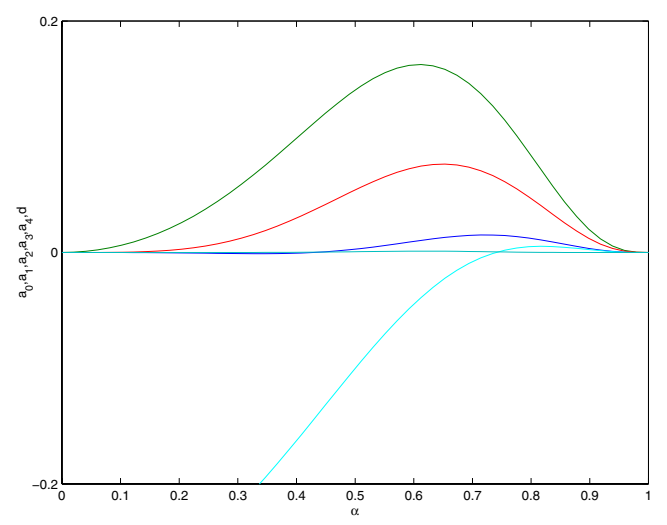

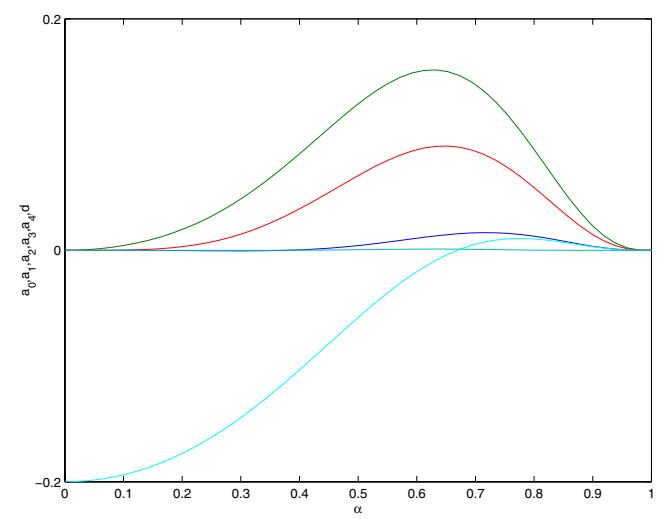

Fig. 12 Plot of the coefficients of the quartic in w (34) for $\mu=$ 1.0, $\operatorname{Fr}^{-1}=0.2, \sigma=1.0$, and variable $\alpha$.

Fig. 13 Plot of the coefficients of the quartic in w (34) for $\mu=1.0, \mathrm{Fr}^{-1}=0.3$, $\sigma=1.0$, and variable $\alpha$.

Theorem 5.2 (Liénard and Chipart criterion) Necessary and sufficient conditions for all the roots of $a_{0} w^{4}+a_{1} w^{3}+$ $a_{2} w^{2}+a_{3} w+a_{4}=0$ to have negative real parts is given by:

$$
a_{0}>0, \quad a_{1}>0, \quad a_{2}>0, \quad a_{3}>0, \quad a_{4}>0, \quad d=a_{1} a_{2} a_{3}-a_{0} a_{3}^{2}-a_{4}, \quad a_{1}^{2}>0 .
$$

Figs. 11, 12 and 13 show the Hurwitz coefficients and Hurwitz determinant of order $3, d$, for the cases $\mathrm{Fr}^{-1}=0.1$, $\sigma=1,1.5$, and $\mu=1.0$, and variable $\alpha$. Comparing these figures with the linear stability plots for the nonrisen and risen relative equilibria Fig. 8 and 5, one observes that there are no other extrema when the non-inverted state is asymptotically unstable and the inverted state is asymptotically stable. In particular, for $\mathrm{Fr}^{-1}=0.1$, observe that there are no other extrema in the range: $0.56<\alpha<1.00$. By evaluating the energy at the non-risen and risen states, in these parameter ranges, the risen and non-risen states are absolute minima and maxima of the constrained energy respectively.

In this regime of parameter values and assuming the Jellett momentum map is constant, the risen state is the only point that can stay identically in $\aleph$ for all time. By the LaSalle invariance principle, the risen state is globally asymptotically stable in the parameter ranges where the only extrema are the risen and non-risen states. This implies the existence of a global connection between the risen and non-risen states if the Jellett momentum map is constant. But, since the Jellett momentum map is not precisely constant, the orbit on the Jellett momentum map approximates the actual global connection with $O\left(\epsilon^{2}\right)$ error on a time-scale $t / \epsilon$ as discussed earlier. Thus, the asymptotic risen state which the Jellett momentum map predicts is $O\left(\epsilon^{2}\right)$ away from the actual asymptotic state, and therefore, within the region of attraction of the actual asymptotic state. Thus, the egg rises all the way when the risen state is stable.

This statement is consistent with the linear stability criteria (21) and (29). In particular, if $h_{4}>0 \Longrightarrow h_{2}<0$, i.e., if the risen state is asymptotically stable the non-risen state is unstable since stability of the risen state implies instability of the non-risen state (cf. Sect. 4).

Outside this range of parameter values, two things can happen: 1) the non-risen state is asymptotically unstable and the risen state is asymptotically unstable; a limit cycle known as a tumbling solution minimizes the energy 2) the spheroid cannot rise to a position that minimizes energy due to its low aspect ratio. In the latter case, the spheroid wobbles like a rattleback 


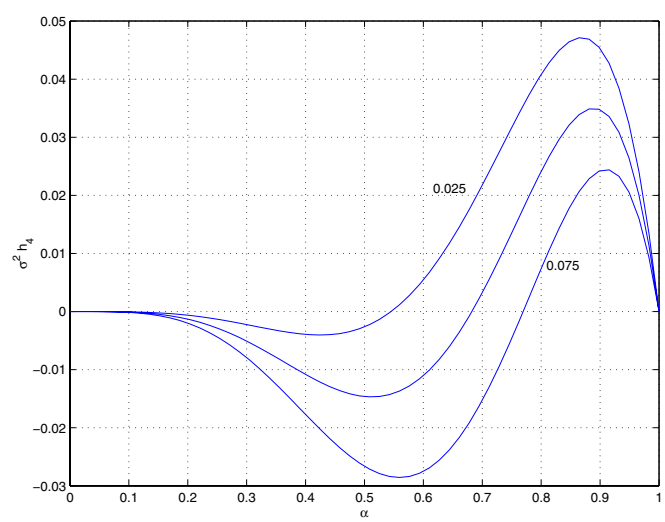

Fig. 14 Plot of $\sigma^{2} h_{4}$ for $\mu=1.0, \mathrm{Fr}^{-1}=0.1,0.2,0.3, \sigma=2 \alpha^{2} /\left(\alpha^{2}+1\right)$, and variable $\alpha$.

and eventually stops or tumbles (See Sect.7.). The Jellett momentum map is not an adiabatic invariant in this low aspect ratio limit, and thus, the results of this section do not directly apply to this case.

The correspondence between the linear and nonlinear results is more profound. Observe that the Hurwitz coefficients which dictate stability of the polynomial in $z$, and hence determine the allowable nutation angles of extrema, is $a_{4}$. The appendix provides an explicit expression for this term. Comparing the expressions for $a_{4}$ with the stability criteria for the rising egg modified Maxwell-Bloch system (29), observe that $h_{4}$ and $a_{4}$ are constant multiples of one another.

\section{Simulation}

For the sake of simulation, suppose the mass distribution in the spheroid is homogeneous with mass $M$ and polar radius $b$. This implies the principal moments of inertia about the axis of symmetry, $I_{k}$, and the equatorial axes, $I$, of the spheroid are given by,

$$
I=\frac{1}{5} M\left(1+\alpha^{2}\right) b^{2}, \quad I_{k}=\frac{2}{5} M \alpha^{2} b^{2} .
$$

From (7), therefore,

$$
\sigma=\frac{I_{k}}{I}=\frac{2 \alpha^{2}}{\alpha^{2}+1}, \quad \mu=\frac{M b^{2}}{I}=\frac{5}{\alpha^{2}+1}, \quad \mathrm{Fr}^{-1}=\frac{g}{\Omega^{2} b} .
$$

Suppose further that $b=0.05 \mathrm{~m}$ and $g=9.8 \mathrm{~m} / \mathrm{s}^{2}$. Using Fig. 14, specific values of $\Omega$ and $\alpha$ are then chosen to simulate rising and tumbling at low Froude number $\left(\mathrm{Fr}^{-1}=0.075\right)$, moderate Froude number $\left(\mathrm{Fr}^{-1}=0.05\right)$, and high Froude number $\left(\mathrm{Fr}^{-1}=0.025\right)$. Friction is valued at $\nu=0.1$ and initial conditions for rising and tumbling with the axis of symmetry pointing up and down respectively are: $\Upsilon_{x}=\Upsilon_{y}=0, \Upsilon_{z}=1.0, l=0.9975, n= \pm 0.05, m=0$, and $x=y=\dot{x}=\dot{y}=0$.

For rising, the aspect ratios $\alpha$ are chosen so that the non-risen state is asymptotically unstable and the risen state is asymptotically stable. These values of $\alpha$ are determined from Fig. 14. For rising at low, moderate, and high Froude number, we pick $\alpha=0.6,0.7,0.8$ respectively. Figs. 15, 16, and 17 show trajectories of the axis of symmetry on the unit ball for rising with the axis of symmetry pointing up and down on the same phase space but different initial conditions.

Similiarly, for tumbling, the aspect ratio $\alpha=0.5$ so that the non-risen and risen states are asymptotically unstable. Figs. 18, 19, and 20 show trajectories of the axis of symmetry on the unit ball for tumbling with the axis of symmetry pointing up and down in the same phase space but different initial conditions. Notice as the Froude number increases, the egg rises or tumbles more rapidly as expected.

The simulations can be downloaded from http://www.acm.caltech.edu/ nawaf/risingegg.html.

\section{Rattleback phenomenon}

The rattleback phenomenon is a very interesting related problem in classical mechanics. A geometric form of the rattleback top is a truncated ellipsoid with an asymmetric mass distribution. Because of this asymmetric mass distribution and dissipation, the rattleback top spins stably about its short axis in one direction, and unstably in the other direction. When spun on a dry surface in the unstable direction, the rattleback top rocks about its intermediate axis and then reverses spin direction as shown in Fig. 21. Moreover, if set in a rocking motion about its intermediate or long axis, the rattleback top tends to spin in the stable direction. Some rattleback tops exhibit multiple spin-reversal which implies that both spin directions about the 

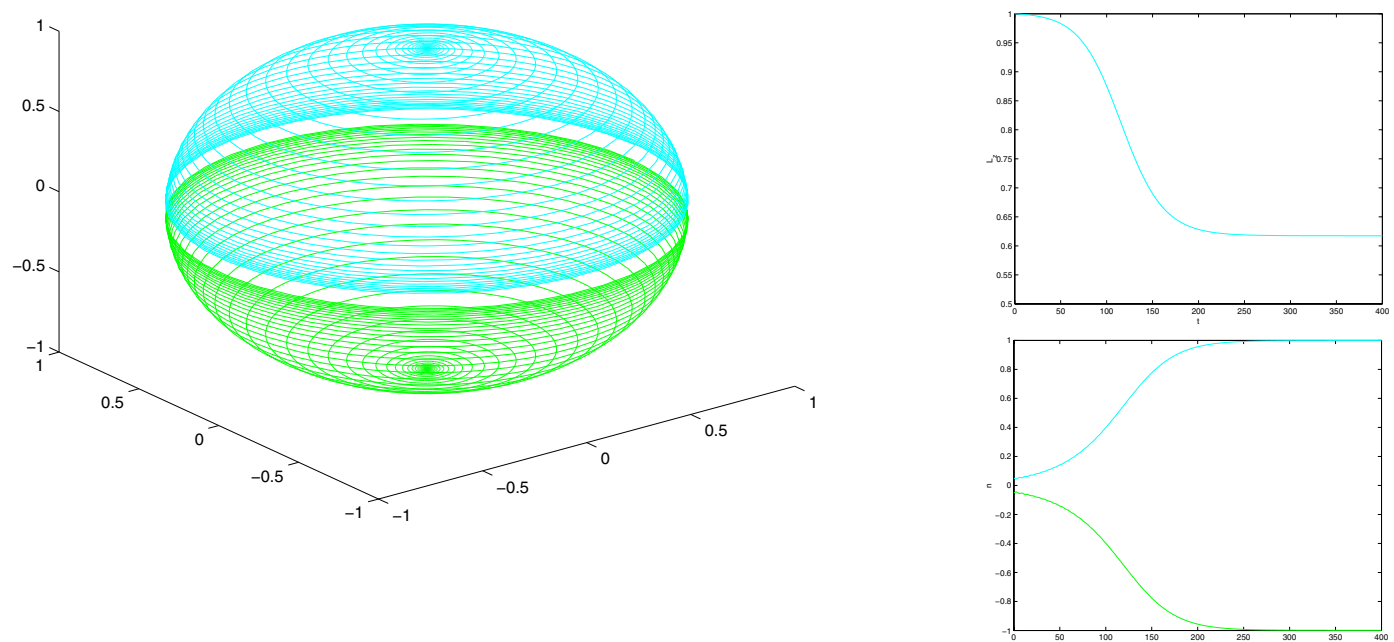

Fig. 15 [Rising at High Froude Number] This figure shows the trajectory of the axis of symmetry for two orbits starting from $n^{0}= \pm 0.05, \Upsilon_{x}^{0}=\Upsilon_{y}^{0}=0, l^{0}=0.9975, m^{0}=0, x^{0}=y^{0}=\dot{x}^{0}=\dot{y}^{0}=0$ for parameters valued at $\mathrm{Fr}^{-1}=$ $0.025, \alpha=0.6, \sigma=0.5294, \mu=3.6765, \nu=0.1$.
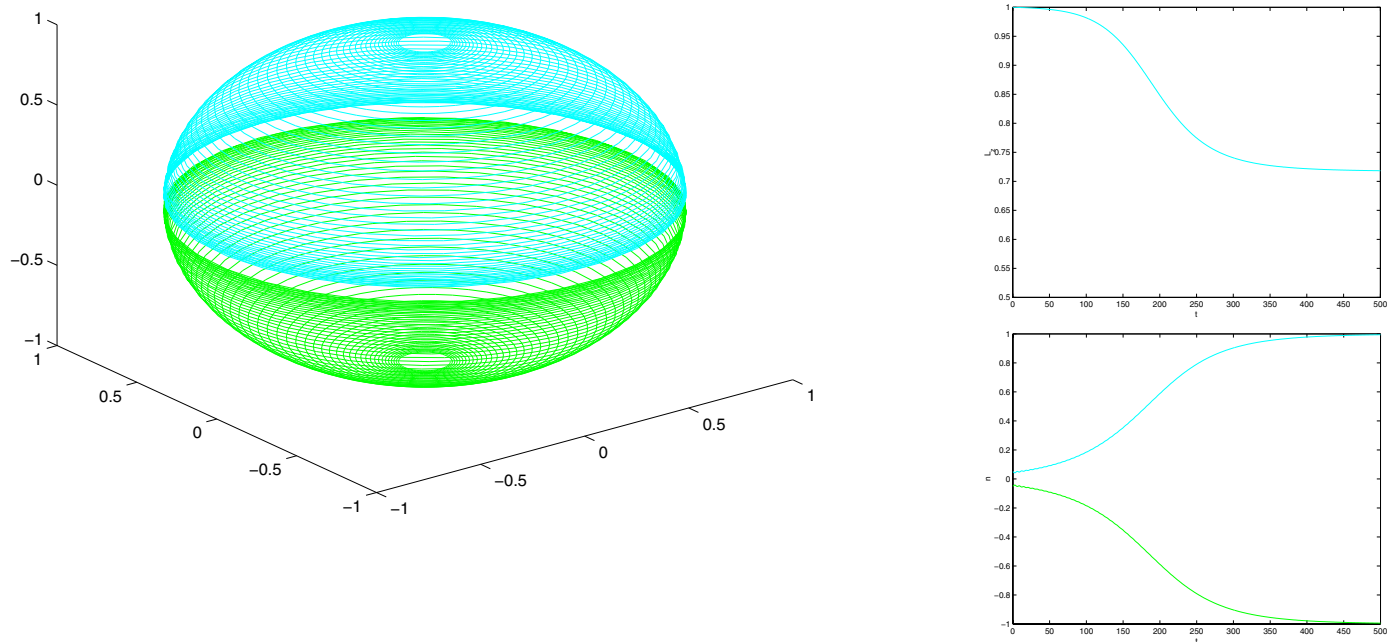

Fig. 16 [Rising at Moderate Froude Number] This figure shows the trajectory of the axis of symmetry for two orbits starting from $n^{0}= \pm 0.05, \Upsilon_{x}^{0}=\Upsilon_{y}^{0}=0, l^{0}=0.9975, m^{0}=0, x^{0}=y^{0}=\dot{x}^{0}=\dot{y}^{0}=0$ for parameters valued at $\mathrm{Fr}^{-1}=0.05, \alpha=0.7, \sigma=0.6577, \mu=3.3557, \nu=0.1$.

short axis are unstable. Because of its asymmetry and dissipation, the rattleback top has no conserved quantity or known adiabatic invariant.

Many scholars in applied math and mechanics have attempted to explain the spin-reversal of the rattleback top. However, the global aspects of the rattleback top are yet to be clarified because the problem challenges existing global dynamical systems theory and tools in novel ways (Hermans [9]). In the literature the rattleback top is modelled as an ellipsoid with crooked principal axes of interia, that is, the axes of inertia are not aligned with the geometrical axes of the ellipsoid as shown in Fig. 22. This geometry suggests a comparison to the rising of an egg.

However, there are some key differences between the egg and the rattleback top. Initially and as it rises, the egg is rapidly spinning. Thus, one can reasonably neglect static friction and assume a sliding friction law. On the other hand, the rattleback top is not in a rapidly spinning state during reversal. This difference becomes more apparent when an egg and rattleback top are spun on an oiled surface: the rapidly spinning egg rises while the rattleback top spins stably in both directions and does not rock. Therefore, static friction is essential to the curious behavior of the rattleback top. In fact, the problem has for a long time been considered a nonholonomic mechanics problem (infinite static friction at point of contact), see Zenkov, Bloch, and Marsden [20] for background. In addition, the rattleback top is an asymmetric top, whereas the egg can be approximated as an axisymmetric body. The rattleback top's asymmetry destroys the adiabatic invariance of the Jellett momentum map. 

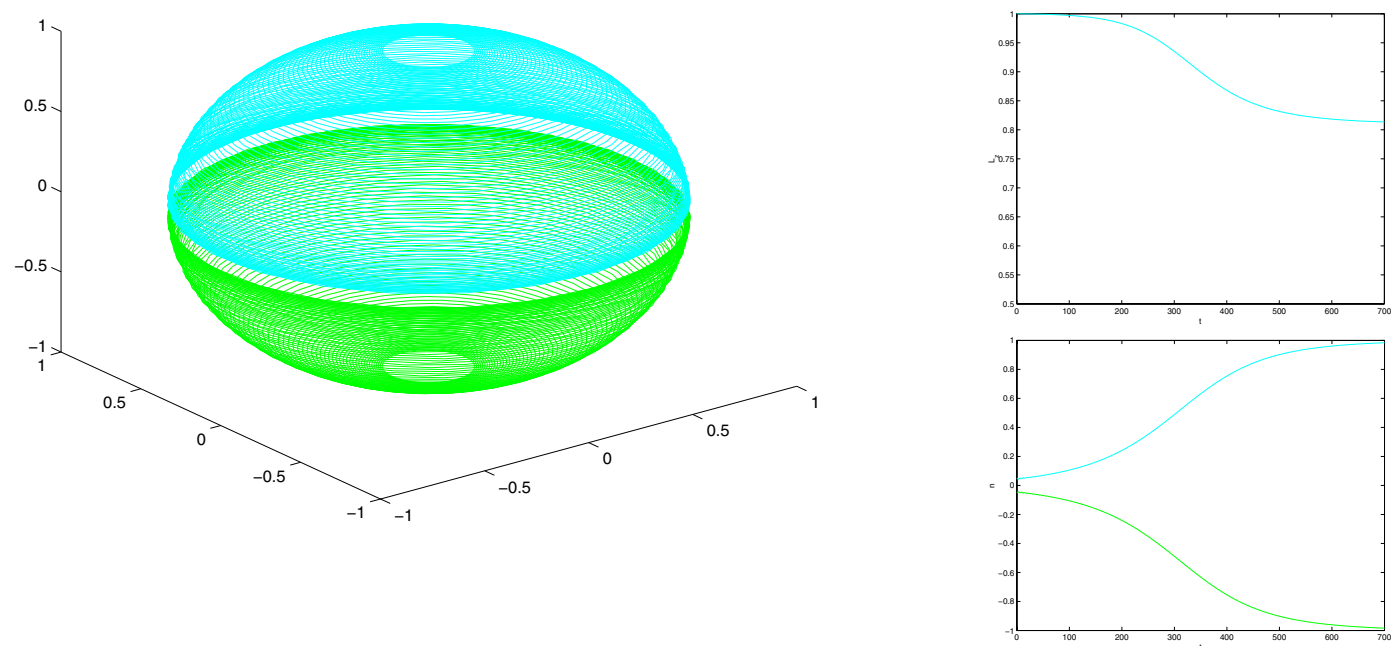

Fig. 17 [Rising at Low Froude Number] This figure shows the trajectory of the axis of symmetry for two orbits starting from $n^{0}= \pm 0.05, \Upsilon_{x}^{0}=\Upsilon_{y}^{0}=0, l^{0}=0.9975, m^{0}=0, x^{0}=y^{0}=\dot{x}^{0}=\dot{y}^{0}=0$ for parameters valued at $\mathrm{Fr}^{-1}=$ $0.075, \alpha=0.8, \sigma=0.7805, \mu=3.0488, \nu=0.1$.
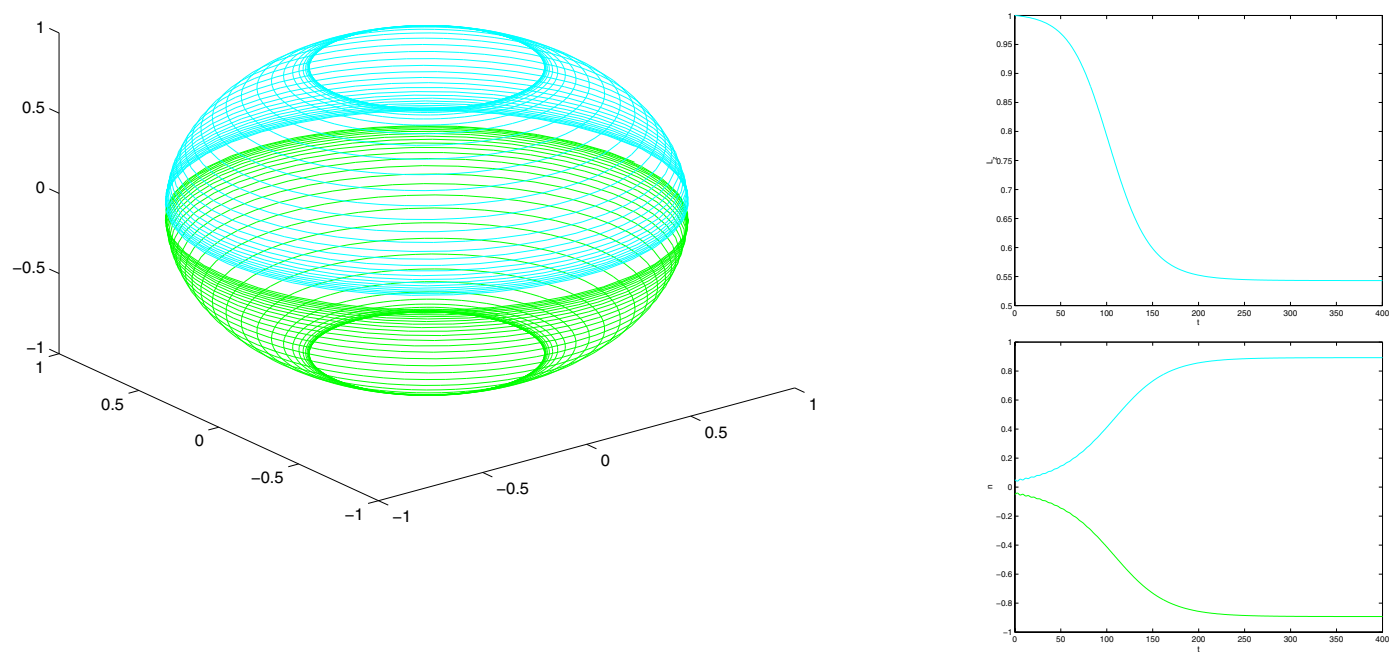

Fig. 18 [Tumbling at High Froude Number] This figure shows the trajectory of the axis of symmetry for two orbits starting from $n^{0}= \pm 0.05, \Upsilon_{x}^{0}=\Upsilon_{y}^{0}=0, l^{0}=0.9975, m^{0}=0, x^{0}=y^{0}=\dot{x}^{0}=\dot{y}^{0}=0$ for parameters valued at $\mathrm{Fr}^{-1}=0.025, \alpha=0.5, \sigma=0.4, \mu=4, \nu=0.1$.

\section{Conclusion}

As mentioned in the introduction, the key difference between the rising of an egg and the rising of a tippe top is the ellipticity of the egg. However, this ellipticity does not fundamentally change the mechanism behind rising. In general, the main result of this paper suggests that tops rise because the potentially unfavorable risen state minimizes total energy subject to an adiabatic momentum constraint; it is through this constraint that rotational kinetic energy is transferred to gravitational potential energy.

The paper mathematically proves this result for the case of an egg modelled as a spheroid under the assumption of a sliding friction law. The conditions for the existence of a heteroclinic connection between the non-risen and risen states of the egg are derived from the theory of dissipation-induced instabilities, LaSalle's invariance principle, and the principle of adiabatic invariance.

The paper shows that the theory of dissipation-induced instabilities (where dissipation is understood as being an energy decreasing non-conservative force) explains the asymptotic instability of the non-risen state and the asymptotic stability of the risen state of the egg. LaSalle's invariance principle determines all of the global asymptotic states of the egg. With the 

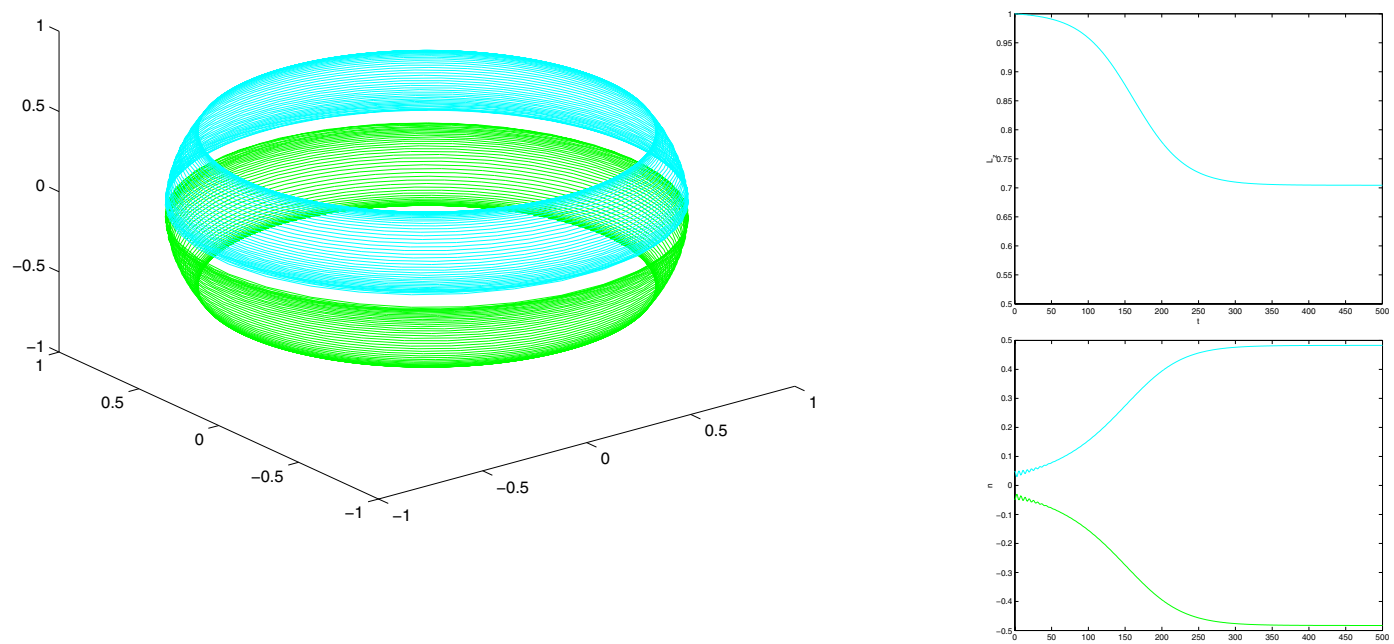

Fig. 19 [Tumbling at Moderate Froude Number] This figure shows the trajectory of the axis of symmetry for two orbits starting from $n^{0}= \pm 0.05, \Upsilon_{x}^{0}=\Upsilon_{y}^{0}=0, l^{0}=0.9975, m^{0}=0, x^{0}=y^{0}=\dot{x}^{0}=\dot{y}^{0}=0$ for parameters valued at $\mathrm{Fr}^{-1}=0.05, \alpha=0.5, \sigma=0.4, \mu=4, \nu=0.1$.
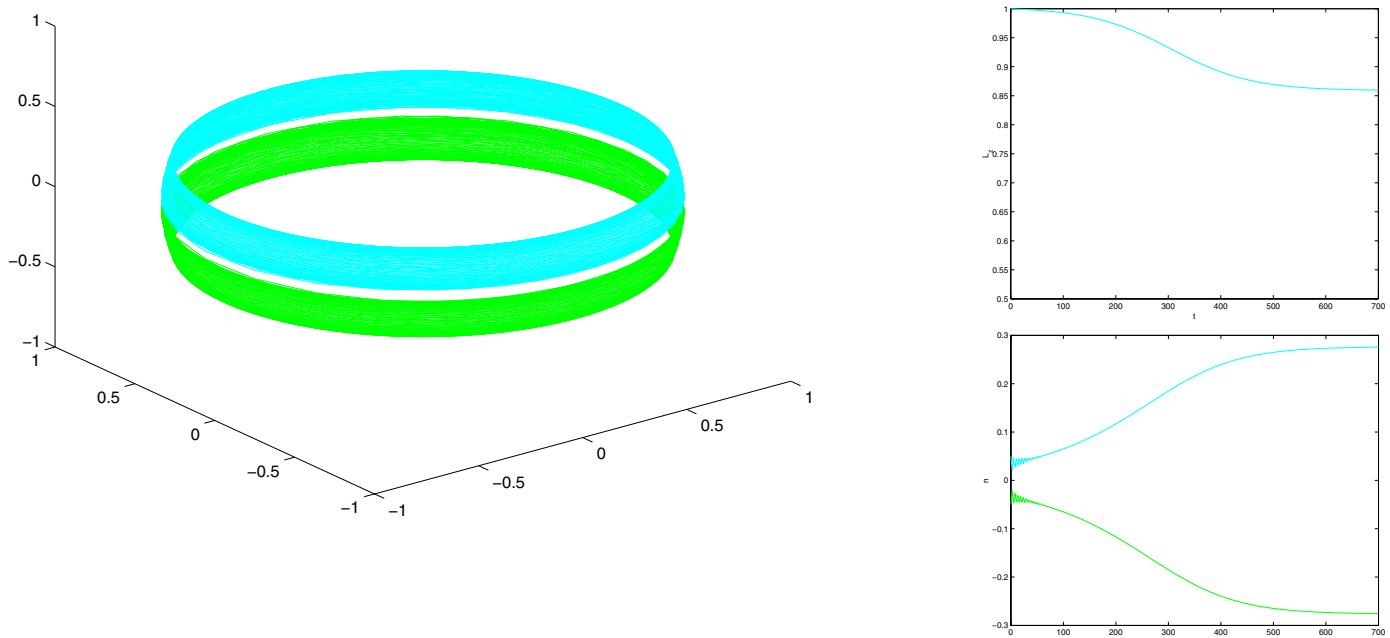

Fig. 20 [Tumbling at Low Froude Number] This figure shows the trajectory of the axis of symmetry for two orbits starting from $n^{0}= \pm 0.05, \Upsilon_{x}^{0}=\Upsilon_{y}^{0}=0, l^{0}=0.9975, m^{0}=0, x^{0}=y^{0}=\dot{x}^{0}=\dot{y}^{0}=0$ for parameters valued at $\mathrm{Fr}^{-1}=0.075, \alpha=0.5, \sigma=0.4, \mu=4, \nu=0.1$.
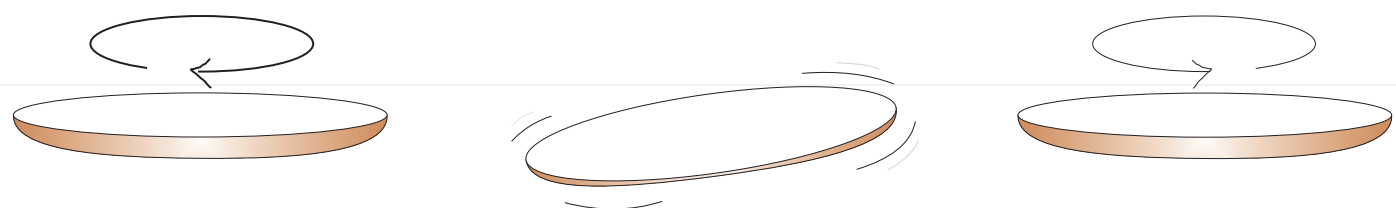

Fig. 21 L-R: Sketch of the rattleback top initially spinning in the unstable direction, rocking back and forth, and then spinning in the opposite direction.

additional requirement that these states minimize the energy subject to the constraint of constant Jellett momentum map, this set of global asymptotic states narrows down to the risen and non-risen states whenever the risen state is asymptotically stable.

Moreover, it is a general theorem (due to Bloch, Krishnaprasad, Marsden, and Ratiu [2]) that any dissipation law - any law that causes the rate of change of energy to be less than zero in the "rising direction" - will move at least one pair of eigenvalues in the unstable direction if one starts with an energetic saddle point (as is the case for gyroscopic systems, like the tippe top or the egg). Thus, the theory should work for other choices of dissipation law as well. 


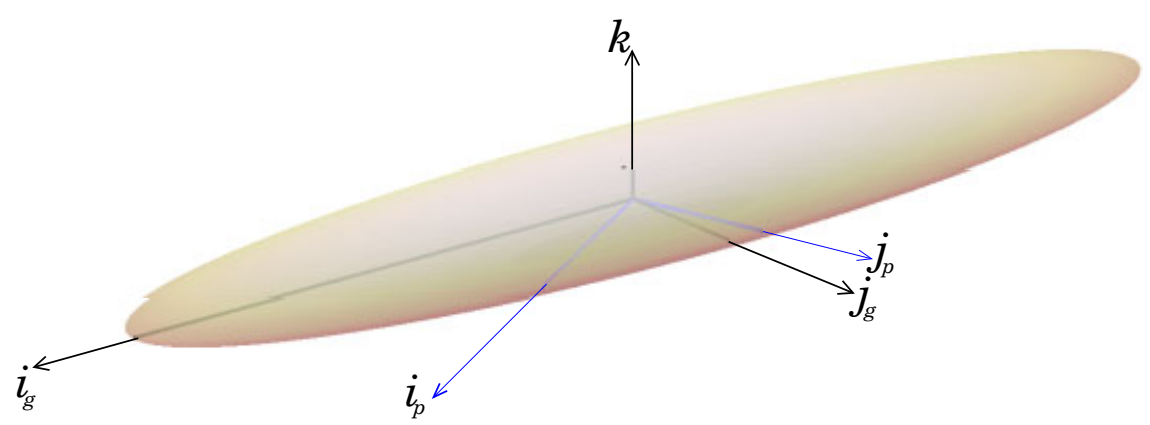

Fig. 22 Typically the rattleback top is modelled as an ellipsoid with crooked principal axes. In this example the principal axes $\mathbf{i}_{p}, \mathbf{j}_{p}$ (blue) are the geometrical axes $\mathbf{i}_{g}, \mathbf{j}_{g}$ (black) rotated about $\mathbf{k}=\mathbf{k}_{p}=\mathbf{k}_{g}$ in the counterclockwise direction by $\pi / 8$.

The procedure to minimize energy subject to an adiabatic momentum constraint is a general nonlinear stability tool which the paper refers to as the energy-adiabatic momentum method.

\section{Appendix}

Normal reaction force. An explicit expression for the surface normal reaction force follows,

$$
\begin{aligned}
& \beta_{1}=-\frac{1}{\sqrt{\alpha^{2}+\left(1-\alpha^{2}\right) n^{2}}}, \\
& f_{z}=\mu \frac{-\beta_{1}^{3} \alpha^{2}\left(1-\alpha^{2}\right) \dot{n}^{2}-\beta_{1} n\left(\dot{m} \Upsilon_{x}-i \Upsilon_{y}\right)\left(1-\alpha^{2}\right)+n\left(l f_{x}+m f_{y}\right)\left(1-\alpha^{2}\right)+\mathrm{Fr}^{-1}}{1+\mu \beta_{1}^{2} n^{2}\left(1-\alpha^{2}\right)^{2}\left(1-n^{2}\right)} .
\end{aligned}
$$

Governing equations in component form. The attitude equations are:

$$
\begin{aligned}
\dot{l} & =\left(-\Upsilon_{z} m+\Upsilon_{y} n\right), \\
\dot{m} & =\left(\Upsilon_{z} l-\Upsilon_{x} n\right), \\
\dot{n} & =\left(-\Upsilon_{y} l+\Upsilon_{x} m\right) .
\end{aligned}
$$

The translational equations are:

$$
\begin{aligned}
\beta_{1} & =-\frac{1}{\sqrt{\alpha^{2}+\left(1-\alpha^{2}\right) n^{2}}}, \\
\ddot{x} & =\frac{f_{x}}{\mu}=-\frac{\nu}{\mu}\left(\dot{x}+\beta_{1} \alpha^{2}\left(\Upsilon_{y}+\frac{1-\sigma}{\sigma}(\mathbf{\Upsilon} \cdot \mathbf{k}) m\right)+\beta_{1}\left(1-\alpha^{2}\right) n \dot{l}\right), \\
\ddot{y} & =\frac{f_{y}}{\mu}=-\frac{\nu}{\mu}\left(\dot{y}-\beta_{1} \alpha^{2}\left(\Upsilon_{x}+\frac{1-\sigma}{\sigma}(\mathbf{\Upsilon} \cdot \mathbf{k}) l\right)+\beta_{1}\left(1-\alpha^{2}\right) n \dot{m}\right) .
\end{aligned}
$$

The rotational equations are:

$$
\begin{aligned}
& \dot{\Upsilon}_{x}=-\frac{f_{y}}{\beta_{1}}+\beta_{1} f_{z}\left(1-\alpha^{2}\right) n m, \\
& \dot{\Upsilon}_{y}=\frac{f_{x}}{\beta_{1}}-\beta_{1} f_{z}\left(1-\alpha^{2}\right) n l, \\
& \dot{\Upsilon}_{z}=\left(1-\alpha^{2}\right) n \beta_{1}\left(-m f_{x}+l f_{y}\right) .
\end{aligned}
$$


Hurwitz coefficients. The values of the parameters in (34) are

$$
\begin{aligned}
& a_{0}=\alpha^{4}\left(1-\alpha^{2}\right)^{2}\left(\alpha^{2}\left(\sigma-\alpha^{2}\right)+\operatorname{Fr}^{-1}\left(-1+\alpha^{2}\right) \mu\left(\alpha^{2}+\alpha^{4}-\sigma\right)^{2}\right), \\
& a_{1}=\alpha^{4}\left(1-\alpha^{2}\right)^{2}\left(\left(1+3 \alpha^{2}\right)\left(\sigma-\alpha^{2}\right)+4 \mu \operatorname{Fr}^{-1} \alpha^{2}\left(\alpha^{6}-\alpha^{2} \sigma+\left(\sigma-\alpha^{2}\right)\right)\right), \\
& a_{2}=\alpha^{2}\left(1-\alpha^{2}\right)^{2}\left(3 \alpha^{2}\left(\sigma-\alpha^{2}\right)\left(\alpha^{2}+1\right)+2 \mu \operatorname{Fr}^{-1}\left(2 \alpha^{8}+\alpha^{6}(-2+\sigma)+\sigma^{2}-\alpha^{2} \sigma(1+\sigma)\right)\right), \\
& a_{3}=\alpha^{4}\left(1-\alpha^{2}\right)^{2}\left(\sigma-\alpha^{2}\right)\left(3+\alpha^{2}-4 \operatorname{Fr}^{-1} \mu\right), \\
& a_{4}=\left(1-\alpha^{2}\right)^{2}\left(\alpha^{4}\left(\sigma-\alpha^{2}\right)-\sigma^{2} \operatorname{Fr}^{-1}\left(1-\alpha^{2}\right) \mu\right) .
\end{aligned}
$$

Geometries that preserve the Jellett momentum map. In the absence of dissipation, the motion of an axisymmetric body on a surface admits two conserved quantities:

$$
P=\mathbf{\Upsilon} \cdot \mathbf{k}, \quad Q=\mathbf{\Upsilon} \cdot \mathbf{e}_{\mathbf{z}} .
$$

In fact, any real-valued function of $P$ and $Q, F(P, Q)$, will also be conserved. With dissipation, the invariant will be of this form, but $F$ will no longer be arbitrary. The Jellett invariant in tippe top inversion is an example of this.

The Jellett momentum map, I, is defined as the Hamiltonian which arises from the $\mathbb{S}^{1}$ action on the configuration space $Q=\mathrm{SO}(3) \times \mathbb{R}^{2}$ (cf. Bou-Rabee et al. [4]). For $\theta \in \mathbb{S}^{1}$, the action corresponds to concurrent rotation about the axis of symmetry by $a \theta$ and the vertical by $b \theta$ where $a$ and $b$ are constants. This action induces the following vector field,

$$
\Psi(y)=a \mathbf{k} \times y+b \mathbf{e}_{\mathbf{z}} \times y .
$$

The corresponding Hamiltonian is given by

$$
I(P, Q)=a P+b Q .
$$

By this symmetry argument, the Jellett momentum map in its most general form is a linear combination of the angular momentum projected on the axis of symmetry and vertical. With dissipation, this momentum map persists as a conservation law if the frictional force at the point of contact does no virtual work under this action.

If the body geometry is spherical, then $\mathbf{q}=a \mathbf{e}_{\mathbf{z}}+b \mathbf{k}$. In this case a direct calculation will show that $\mathbf{I}=\mathbf{\Upsilon} \cdot \mathbf{q}$ is conserved.

Lemma 9.1 An axisymmetric body conserves the Jellett momentum map if and only if the contact vector has the form:

$$
\mathbf{q}=\alpha(n)\left(c \mathbf{e}_{\mathbf{z}}+\mathbf{k}\right)
$$

where $c$ is a constant.

From (8) it is clear that $\dot{\Upsilon} \cdot \mathbf{q}=0$, and therefore,

$$
\alpha(n) \dot{Q}+\beta(n) \dot{P}=0 \Longrightarrow \dot{P}=-\frac{\alpha(n)}{\beta(n)} \dot{Q} .
$$

Because $\beth=\Xi(P, Q)$, by (35), the necessary and sufficient condition for $\beth$ to be conserved is that,

$$
\dot{\beth}=\frac{\partial \beth}{\partial P} \dot{P}+\frac{\partial \beth}{\partial Q} \dot{Q}=0
$$

And since $\dot{P}=-\alpha(n) / \beta(n) \dot{Q}$ we have,

$$
\dot{\beth}=\left(\frac{\partial \beth}{\partial Q}-\frac{\alpha(n)}{\beta(n)} \frac{\partial \beth}{\partial P}\right) \dot{Q}
$$

which equals zero if and only if $\alpha(n) / \beta(n)=$ constant $=c$ since $\mathrm{I}$ is a linear combination of $P$ and $Q$ (cf. (35)).

Lemma 9.2 If the contact vector has the form,

$$
\mathbf{q}=\alpha(n)\left(c \mathbf{e}_{\mathbf{z}}+\mathbf{k}\right)
$$

then the most general form of the corresponding body is a displaced sphere. 


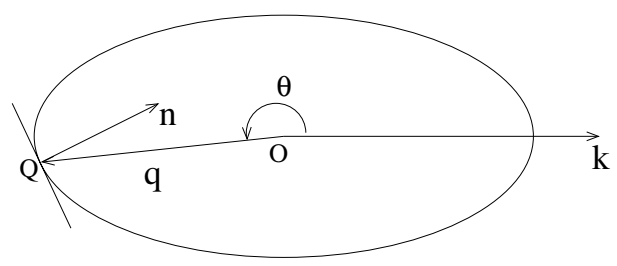

Fig. 23 The reference frame shown is used to determine the most general surface corresponding to a contact vector of the form $\mathbf{q}=\gamma(\theta)\left(c \mathbf{e}_{\mathbf{z}}+\mathbf{k}\right)$.

Because of axisymmetry it suffices to consider just a cross-section of the surface of rotation. This planar surface is deemed fixed, i.e., $\mathbf{k}=(1,0)$ and the contact vector $\mathbf{q}$ at an angle $\theta$ with $\mathbf{k}$ as shown in Fig. 23.

In the coordinates of (8) $\cos \theta=n$, i.e., $\theta$ is the nutation angle. The contact vector, $\mathbf{q}$, can be expressed in polar form as:

$$
\mathbf{q}=r(\theta)(\cos (\theta), \sin (\theta))=r(\theta) \mathbf{a}(\theta)
$$

with tangent vector:

$$
\mathbf{t}_{q}=r^{\prime}(\theta) \mathbf{a}(\theta)+r(\theta) \mathbf{a}^{\prime}(\theta)
$$

and unit normal vector:

$$
\mathbf{u n}_{q}=\frac{1}{\sqrt{\left(r^{\prime}\right)^{2}+r^{2}}}\left(r(\theta) \mathbf{a}^{\prime}(\theta)-r^{\prime}(\theta) \mathbf{a}(\theta)\right) .
$$

In these coordinates eq. (36) can be expressed as,

$$
\mathbf{q}=\gamma(\theta)\left(c \mathbf{u n}_{q}+(1,0)\right)=r(\theta) \mathbf{a}(\theta)
$$

which can be rewritten as,

$$
\gamma(\theta)\left(-c \frac{r(\theta)}{\sqrt{\left(r^{\prime}\right)^{2}+r^{2}}}+\cos \theta-r(\theta)\right) \mathbf{a}(\theta)+\gamma(\theta)\left(-c \frac{r^{\prime}(\theta)}{\sqrt{\left(r^{\prime}\right)^{2}+r^{2}}}+\sin \theta\right) \mathbf{a}^{\prime}(\theta)=0 .
$$

Since $\mathbf{a}$ and $\mathbf{a}^{\prime}$ are linearly independent, this equation is satisfied if and only if

$$
\begin{aligned}
\gamma(\theta)\left(-c \frac{r(\theta)}{\sqrt{\left(r^{\prime}\right)^{2}+r^{2}}}+\cos \theta-r(\theta)\right) & =0, \\
\gamma(\theta)\left(-c \frac{r^{\prime}(\theta)}{\sqrt{\left(r^{\prime}\right)^{2}+r^{2}}}+\sin \theta\right) & =0 .
\end{aligned}
$$

The second equation implies

$$
r^{\prime}(\theta)= \pm \frac{\sin \theta r}{\sqrt{c^{2}-\sin \theta^{2}}}
$$

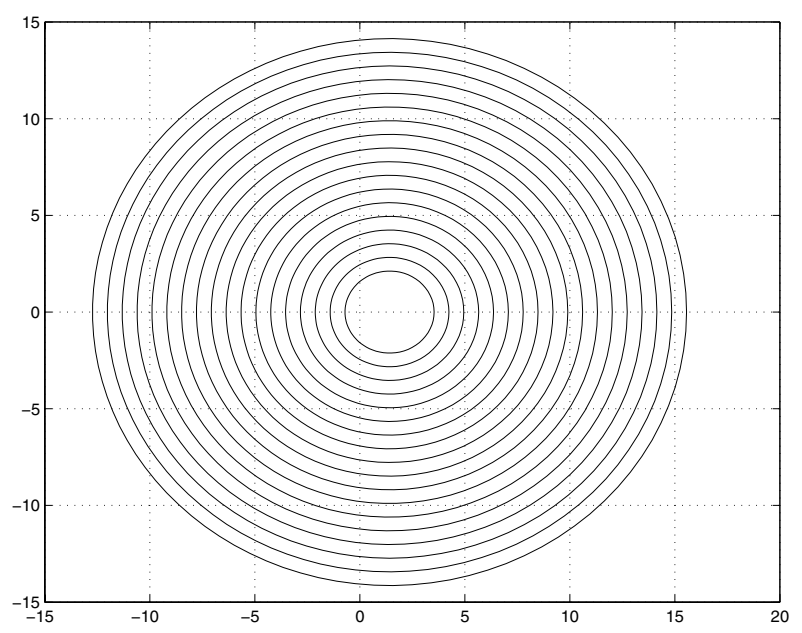

Fig. 24 If $\mathbf{q}=\gamma(\theta)\left(c \mathbf{e}_{\mathbf{z}}+\mathbf{k}\right)$, then the contact vector parametrizes displaced circles in the plane as shown above for $1<c \leq 10$. 
or that,

$$
r(\theta)=c_{1}\left(\sqrt{2} \cos \theta+\sqrt{-1+2 c^{2}+\cos 2 \theta}\right)^{ \pm 1} .
$$

For $c>1$, we compute:

$$
(r(\theta) \cos \theta-a)^{2}+(r(\theta) \sin \theta)^{2}=r(\theta)^{2}-2 a r \cos \theta+a^{2} .
$$

If we pick $a=\sqrt{2}$, this expression reduces to:

$$
r(\theta)^{2}-2 a r \cos \theta+a^{2}=2 c^{2}
$$

which proves that the contact vector parametrizes a displaced circle as shown in Fig. 24. Since the contact vector also parametrizes the cross-section of the body, the corresponding planar curve is circular and in three dimensions a ball.

Therefore,

Proposition 9.3 The Jellett momentum map is a conserved quantity if and only if the body geometry is spherical.

Acknowledgements We wish to acknowledge Darryl Holm, Andy Ruina, Demetri Spanos, and Ahmed Bou-Rabee for helpful remarks. Bou-Rabee's research was supported by the US DOE Computational Science Graduate Fellowship through grant DE-FG02-97ER25308; Marsden's research partially supported by the National Science Foundation through NSF grant DMS-0204474; and Romero's research was supported by Sandia National Laboratories. Sandia is a multiprogram laboratory operated by Sandia Corporation, a Lockheed Martin Company, for the United States Department of Energy under contract DE-AC04-94AL85000. The U.S. Government retains a nonexclusive, royalty-free license to publish or reproduce the published form of this contribution, or allow others to do so, for U.S. Government purposes. Copyright is owned by ZAMM to the extent not limited by these rights.

\section{References}

[1] A. Bloch, Nonholonomic Mechanics and Control, Interdisciplinary Applied Mathematics, Vol. 24 (Springer-Verlag, Berlin, Heidelberg, New York, 2003), pp. 72-74.

[2] A. M. Bloch, P. S. Krishnaprasad, J.E. Marsden, and T. S. Ratiu, Ann. Inst. Henri Poincare Anal. Nonlineaire 11, 37-90 (1994).

[3] A. M. Bloch, P. S. Krishnaprasad, J.E. Marsden, and T. S. Ratiu, Commun. Math. Phys. 175, 1-42 (1996).

[4] N.M. Bou-Rabee, J.E. Marsden, and L.A. Romero, SIADS 3, 352-377 (2004).

[5] H. Crabtree, Spinning Tops and Gyroscopic Motions. Chelsea, New York, 147-155 (1909).

[6] F. R. Gantmacher, The Theory of Matrices, Vol. II (Chelsea, New York, 1959), pp. 190-196, 220-225.

[7] S. M. Jalnapurkar and J.E. Marsden, Regul. Chaotic Dyn. 3, 161-179 (1999).

[8] R. M. Harrigan, US Patent No. 4,382,245 (1983).

[9] J. Hermans, Rolling Rigid Bodies with and without Symmetries, Ph.D. thesis, University of Utrecht (1995).

[10] E. W. Hones and W. G. Hones, US Patent No. 5,883,454 (1999).

[11] J.H. Jellett, A Treatise on the Theory of Friction (Macmillan, London, 1872).

[12] J.E. Marsden and T. S. Ratiu, Introduction to Mechanics and Symmetry, Second Ed. (Springer-Verlag, New York, 1999), pp. 383396.

[13] H. K. Moffatt and Y. Shimomura, Nature 416, 385-386 (2002).

[14] H. K. Moffatt, Y. Shimomura, and M. Branicki, Proc. R. Soc. A, to appear (2004).

[15] Y. Shiomura, M. Branicki, and H. K. Moffatt, Proc. R. Soc. A, to appear (2004).

[16] E. J. Routh, The Advanced Part of a Treatise on the Dynamics of a System of Rigid Bodies (Macmillan, New York, 1905), pp. 195-196.

[17] A.L. Ruina, Rolling and Sliding of spinning things: Euler's Disk, Jellett's Egg and Moffatt's Nature. Retrieved January 21, 2004, from http: / / tam. cornell. edu/ ruina/hplab/Rolling\%20and\%20sliding/index.html (2002).

[18] K. Sasaki, AJP to appear (2003).

[19] W. Thomson and P. G. Tait, Treatise on Natural Philosophy (Cambridge University Press, Cambridge, 1879).

[20] D. V. Zenkov, A. M. Bloch, and J. E. Marsden, Dyn. Stab. Syst. 13, 123-166 (1998). 